MATHEMATICS OF COMPUTATION

Volume 68, Number 227, Pages 1257-1281

S 0025-5718(99)01047-9

Article electronically published on February 23, 1999

\title{
ELLIPTIC BINOMIAL DIOPHANTINE EQUATIONS
}

\author{
ROELOF J. STROEKER AND BENJAMIN M. M. DE WEGER
}

\begin{abstract}
The complete sets of solutions of the equation $\left(\begin{array}{l}n \\ k\end{array}\right)=\left(\begin{array}{c}m \\ \ell\end{array}\right)$ are determined for the cases $(k, \ell)=(2,3),(2,4),(2,6),(2,8),(3,4),(3,6),(4,6)$, $(4,8)$. In each of these cases the equation is reduced to an elliptic equation, which is solved by using linear forms in elliptic logarithms. In all but one case this is more or less routine, but in the remaining case $((k, \ell)=(3,6))$ we had to devise a new variant of the method.
\end{abstract}

\section{INTRODUCTION}

In Pascal's Triangle, composed of the binomial coefficients $\left(\begin{array}{l}n \\ k\end{array}\right)$ for $n=$ $0,1,2, \ldots, 0 \leq k \leq n$, all natural numbers, with the exception of 2 , occur at least twice, and many three times or more. Not counting multiple occurrences of trivial type, coming from $\left(\begin{array}{l}n \\ 0\end{array}\right)=1,\left(\begin{array}{l}n \\ 1\end{array}\right)=n$ and $\left(\begin{array}{l}n \\ k\end{array}\right)=\left(\begin{array}{c}n \\ n-k\end{array}\right)$, one could formulate the following problem.

Main Problem. To determine all natural numbers that occur at least twice in Pascal's Triangle as binomial coefficient $\left(\begin{array}{l}n \\ k\end{array}\right)$ with $2 \leq k \leq \frac{1}{2} n$.

As yet this problem is unsolved in its full generality. The only nontrivial solutions known at this time are the following:

$$
\begin{gathered}
\left(\begin{array}{c}
16 \\
2
\end{array}\right)=\left(\begin{array}{c}
10 \\
3
\end{array}\right)=120, \quad\left(\begin{array}{c}
21 \\
2
\end{array}\right)=\left(\begin{array}{c}
10 \\
4
\end{array}\right)=210, \quad\left(\begin{array}{c}
56 \\
2
\end{array}\right)=\left(\begin{array}{c}
22 \\
3
\end{array}\right)=1540, \\
\left(\begin{array}{c}
120 \\
2
\end{array}\right)=\left(\begin{array}{c}
36 \\
3
\end{array}\right)=7140, \quad\left(\begin{array}{c}
153 \\
2
\end{array}\right)=\left(\begin{array}{c}
19 \\
5
\end{array}\right)=11628, \quad\left(\begin{array}{c}
221 \\
2
\end{array}\right)=\left(\begin{array}{c}
17 \\
8
\end{array}\right)=24310, \\
\left(\begin{array}{c}
78 \\
2
\end{array}\right)=\left(\begin{array}{c}
15 \\
5
\end{array}\right)=\left(\begin{array}{c}
14 \\
6
\end{array}\right)=3003, \\
\text { and } \quad\left(\begin{array}{c}
F_{2 i+2} F_{2 i+3} \\
F_{2 i} F_{2 i+3}
\end{array}\right)=\left(\begin{array}{c}
F_{2 i+2} F_{2 i+3}-1 \\
F_{2 i} F_{2 i+3}+1
\end{array}\right) \quad \text { for } \quad i=1,2, \ldots,
\end{gathered}
$$

where $F_{n}$ is the $n$th Fibonacci number, defined by $F_{0}=0, F_{1}=1$, and $F_{n+1}=$ $F_{n}+F_{n-1}$ for $n=1,2, \ldots$ This infinite family of solutions is due to D.A. Lind [L] and D.A. Singmaster [Sin]. It is conjectured that there are no other nontrivial solutions, and it is known that there are none with $\left(\begin{array}{l}n \\ k\end{array}\right) \leq 10^{30}$ or $n \leq 1000$, cf. [dW2].

Received by the editor October 16, 1997.

1991 Mathematics Subject Classification. Primary 11D25, 11G05; Secondary 11B65, 14H52.

Key words and phrases. Diophantine equation, elliptic curve, binomial coefficient.

The second author's research was supported by the Netherlands Mathematical Research Foundation SWON with financial aid from the Netherlands Organization for Scientific Research NWO.

(C)1999 American Mathematical Society 
For fixed $k, \ell\left(\right.$ with $2 \leq k<\ell$ ) the equation $\left(\begin{array}{l}n \\ k\end{array}\right)=\left(\begin{array}{c}m \\ \ell\end{array}\right)$ has been completely solved in three cases only, namely for $(k, \ell)=(2,3)$ by È.T. Avanesov [A], for $(k, \ell)=(2,4)$ by one of us [dW1] and independently by A. Pintér $[\mathrm{P}]$, and for $(k, \ell)=(3,4)$ by a remark of one of us, noting that the equation in this case reduces at once to an equation solved by L.J. Mordell [M1], see [dW2].

For a few choices of $(k, \ell)$ the equation $\left(\begin{array}{l}n \\ k\end{array}\right)=\left(\begin{array}{c}m \\ \ell\end{array}\right)$ represents an elliptic curve, or can be transformed into another equation representing an elliptic curve. Note that $\left(\begin{array}{l}n \\ k\end{array}\right)$ is, for fixed $k$, a polynomial in $n$ of degree $k$ with rational coefficients. For even $k$, using the obvious symmetry about $n-\frac{k-1}{2}$, it is also a polynomial in $\left(n-\frac{k-1}{2}\right)^{2}$ of degree $\frac{1}{2} k$ with rational coefficients. In particular,

$\left(\begin{array}{l}n \\ 2\end{array}\right)$ is quadratic in $n, \quad\left(\begin{array}{l}n \\ 3\end{array}\right)$ is cubic in $n$,

$\left(\begin{array}{l}n \\ 4\end{array}\right)$ is quartic in $n$, and also quadratic in $\left(n-\frac{3}{2}\right)^{2}$,

$\left(\begin{array}{l}n \\ 6\end{array}\right)$ is cubic in $\left(n-\frac{5}{2}\right)^{2}, \quad\left(\begin{array}{l}n \\ 8\end{array}\right)$ is quartic in $\left(n-\frac{7}{2}\right)^{2}$.

Now, let $f_{q}, g_{q} \in \mathbb{Z}[x]$ be polynomials of degree $q$. Then the equations

$$
y^{2}=f_{3}(x), \quad y^{2}=f_{4}(x), \quad f_{3}(y)=g_{3}(x)
$$

generically represent elliptic curves. Equations of the first two types $y^{2}=f_{q}(x)$ for $q=3,4$ can be reduced to a number of Thue equations, cf. L.J. Mordell [M1], and for Thue equations general solution methods exist, based on estimations for linear forms in logarithms of algebraic numbers, cf. N. Tzanakis and B.M.M. de Weger $[\mathrm{TW}]$. Such methods were used in $[\mathrm{dW} 1]$ and $[\mathrm{P}]$ cited above, for solving $\left(\begin{array}{l}n \\ 2\end{array}\right)=\left(\begin{array}{c}m \\ 4\end{array}\right)$. An alternative, though often less practical approach is provided by Yu. Bilu and G. Hanrot [BH]. For the case $f_{3}(y)=g_{3}(x)$ however we do not know of any practical method that works in general. Only some very special types can be solved, such as superelliptic equations (where $f_{3}(y)=y^{3}$, see $[\mathrm{BH}]$ ). In theory an upper bound for the absolute values of the coordinates of an integral point on any model $g(x, y)=0$ for an elliptic curve is explicitly known (cf. [BC]), but the known upper bounds are so large as to render the corresponding search range completely unworkable.

Only recently techniques have been developed involving estimations of linear forms in elliptic logarithms for the solution of elliptic diophantine equations. We felt that most likely this method should also work quite efficiently for the binomial equations at hand. Success should be almost guaranteed for any reasonable equation of type $y^{2}=f_{q}(x)$ for $q=3,4$, as the elliptic logarithm method, developed independently by R.J. Stroeker and N. Tzanakis [ST1], by J. Gebel, A. Pethő and H.G. Zimmer [GPZ], and by N.P. Smart [Sm] for $q=3$, and by N. Tzanakis [T] for $q=4$, is quite generally applicable.

For the case $f_{3}(y)=g_{3}(x)$, which also represents an elliptic curve, a similar method should be applicable, but such a method is not to be found yet in the literature. As it turned out, the main ideas of [ST1] and [T] carried through to this case too, without significant changes. This points at the probable existence of an efficient method for solving any reasonable equation of type $f_{3}(y)=g_{3}(x)$, and even of the more general type $f_{3}(x, y)=0$, where $f \in \mathbb{Z}(x, y)$ has degree 3 and is not 
necessarily homogeneous. We study this general type of equation in a forthcoming paper [SW]; in the present paper we merely concentrate on the particular equation coming from $\left(\begin{array}{l}n \\ 3\end{array}\right)=\left(\begin{array}{c}m \\ 6\end{array}\right)$.

All this enabled us to prove the following results. Three of them are already known, but for these we present different proofs. From now on we do not maintain the restriction $m, n>0$ anymore, but we will allow $m, n \in \mathbb{Z}$, based on the definition

$$
\left(\begin{array}{l}
n \\
k
\end{array}\right)=\frac{1}{k !} \prod_{i=0}^{k-1}(n-i) \quad \text { for } k \geq 0 .
$$

Theorem A23 (Avanesov). The only solutions of $\left(\begin{array}{l}n \\ 2\end{array}\right)=\left(\begin{array}{c}m \\ 3\end{array}\right)$ are those listed in Table T23.

Theorem A24 (de Weger, Pintér). The only solutions of $\left(\begin{array}{l}n \\ 2\end{array}\right)=\left(\begin{array}{c}m \\ 4\end{array}\right)$ are those listed in Table T24.

Theorem A26. The only solutions of $\left(\begin{array}{l}n \\ 2\end{array}\right)=\left(\begin{array}{c}m \\ 6\end{array}\right)$ are those listed in Table T26.

Theorem A28. The only solutions of $\left(\begin{array}{l}n \\ 2\end{array}\right)=\left(\begin{array}{c}m \\ 8\end{array}\right)$ are those listed in Table T28.

Theorem A34 (Mordell, de Weger). The only solutions of $\left(\begin{array}{l}n \\ 3\end{array}\right)=\left(\begin{array}{c}m \\ 4\end{array}\right)$ are those listed in Table T34.

Theorem A36. The only solutions of $\left(\begin{array}{l}n \\ 3\end{array}\right)=\left(\begin{array}{c}m \\ 6\end{array}\right)$ are those listed in Table T36.

Theorem A46. The only solutions of $\left(\begin{array}{l}n \\ 4\end{array}\right)=\left(\begin{array}{c}m \\ 6\end{array}\right)$ are those listed in Table T46.

Theorem A48. The only solutions of $\left(\begin{array}{l}n \\ 4\end{array}\right)=\left(\begin{array}{c}m \\ 8\end{array}\right)$ are those listed in Table T48.

One might wonder what can be said about other combinations of values for $k$ and $\ell$ in $\left(\begin{array}{l}n \\ k\end{array}\right)=\left(\begin{array}{l}m \\ \ell\end{array}\right)$ than those considered here. If $k=2$ or $k=4$ then we encounter an equation of type $y^{2}=f_{\ell}(x)$, which represents a hyperelliptic curve of genus $\left\lfloor\frac{\ell-1}{2}\right\rfloor \geq 2$ when $\ell \geq 5$. For such equations there are approaches that should work as a rule, like those by Thue equations, or by Bilu's method [BH], but in any practical sense these techniques seem too complex by far, even for the case of $\left(\begin{array}{l}n \\ 2\end{array}\right)=\left(\begin{array}{c}m \\ 5\end{array}\right)$. For other values of $(k, \ell)$ no methods are known, and all we can say is that in many of these cases the binomial equation $\left(\begin{array}{l}n \\ k\end{array}\right)=\left(\begin{array}{c}m \\ \ell\end{array}\right)$ possibly represents an algebraic curve of genus $>1$, and therefore at most finitely many rational solutions exist, by celebrated results of G. Faltings $[F]$.

Very recently we have been able to completely solve the equations $\left(\begin{array}{l}n \\ k\end{array}\right)=$ $\left(\begin{array}{l}n-1 \\ k+2\end{array}\right)$ (which has no nontrivial solutions) and $\left(\begin{array}{l}n \\ k\end{array}\right)=\left(\begin{array}{l}n-2 \\ k+1\end{array}\right)$ (which has no nontrivial solutions other than $\left(\begin{array}{l}6 \\ 1\end{array}\right)=\left(\begin{array}{l}4 \\ 2\end{array}\right)$ ). We did this by the same variant of the elliptic logarithms method that we use in the present paper for solving $\left(\begin{array}{l}n \\ 3\end{array}\right)=\left(\begin{array}{c}m \\ 6\end{array}\right)$. We intend to give details in our forthcoming paper [SW]. 
TABLE 1. Transformations from binomial equations to elliptic equations

\begin{tabular}{|c|ll|}
\hline$(k, \ell)$ & \multicolumn{2}{|c|}{ transformation } \\
\hline$(2,3)$ & $X=3 m-3$ & $Y=9 n-5$ \\
$(2,4)$ & $U=m-2$ & $V=6 n-3$ \\
$(2,6)$ & $X=\frac{5}{2} m^{2}-\frac{25}{2} m+8$ & $Y=75 n-38$ \\
$(2,8)$ & $U=\frac{1}{2} m^{2}-\frac{7}{2} m+6$ & $V=210 n-105$ \\
$(3,4)$ & $X=n-1$ & $Y=\frac{1}{2} m^{2}-\frac{3}{2} m$ \\
$(3,6)$ & $U=n-1$ & $V=\frac{1}{2} m^{2}-\frac{5}{2} m+3$ \\
$(4,6)$ & $X=\frac{15}{2} m^{2}-\frac{75}{2} m+25$ & $Y=\frac{225}{2} n^{2}-\frac{675}{2} n+112$ \\
$(4,8)$ & $U=\frac{1}{2} m^{2}-\frac{7}{2} m+3$ & $V=105 n^{2}-315 n+105$ \\
\hline
\end{tabular}

We now turn to the above mentioned eight cases, which we shall call elliptic binomial equations. Inspired by the above remarks, we introduce changes of variables as given in Table 1 . Notice that the new variables $(X, Y$ or $U, V)$ are integral valued.

These transformations are chosen such that the resulting equations have a convenient form. For the cases of type $f_{2}(Y)=g_{3}(X)$ this means that a global minimal Weierstrass equation over $\mathbb{Z}$ is obtained; for the cases of type $f_{2}(V)=g_{4}(U)$ this means that $f_{2}(V)=V^{2}$, the constant term in $g_{4}(U)$ is a square, and the coefficients are integers as small as possible; and for the case $f_{3}(U)=g_{3}(V)$ the coefficients of both polynomials are again integers, as small as possible.

It now will be clear that Theorems A23 to A48 are consequences of the following results.

Theorem B23. The complete set of solutions in rational integers $X, Y$ of the equation

$$
Y^{2}+Y=X^{3}-9 X+20
$$

is given in Table $\mathrm{T} 23$.

TABLE T23. The solutions to (W23) and to $\left(\begin{array}{l}n \\ 2\end{array}\right)=\left(\begin{array}{c}m \\ 3\end{array}\right)$

\begin{tabular}{|rr|rr|rr|}
\hline$X$ & $Y$ & $n$ & $m$ & $m_{1}$ & $m_{2}$ \\
\hline-3 & -5 & 0 & 0 & 1 & 1 \\
-3 & 4 & 1 & 0 & -1 & -1 \\
-2 & -6 & & & 0 & -2 \\
-2 & 5 & & & 0 & 2 \\
0 & -5 & 0 & 1 & -1 & 0 \\
0 & 4 & 1 & 1 & 1 & 0 \\
1 & -4 & & & 2 & 0 \\
1 & 3 & & & -2 & 0 \\
3 & -5 & 0 & 2 & 0 & -1 \\
3 & 4 & 1 & 2 & 0 & 1 \\
6 & -14 & -1 & 3 & -1 & 1 \\
\hline
\end{tabular}

\begin{tabular}{|rr|rr|rr|}
\hline$X$ & $Y$ & $n$ & $m$ & $m_{1}$ & $m_{2}$ \\
\hline 6 & 13 & 2 & 3 & 1 & -1 \\
10 & -31 & & & -2 & -2 \\
10 & 30 & & & 2 & 2 \\
12 & -41 & -4 & 5 & 2 & 1 \\
12 & 40 & 5 & 5 & -2 & -1 \\
27 & -140 & -15 & 10 & 1 & -2 \\
27 & 139 & 16 & 10 & -1 & 2 \\
63 & -500 & -55 & 22 & -3 & 0 \\
63 & 499 & 56 & 22 & 3 & 0 \\
105 & -1076 & -119 & 36 & 1 & 3 \\
105 & 1075 & 120 & 36 & -1 & -3 \\
\hline
\end{tabular}

Theorem B24. The complete set of solutions in rational integers $U, V$ of the equation

$$
V^{2}=3 U^{4}+6 U^{3}-3 U^{2}-6 U+9
$$


is given in Table $\mathrm{T} 24$.

TABLE T24. The solutions to (Q24) and to $\left(\begin{array}{l}n \\ 2\end{array}\right)=\left(\begin{array}{c}m \\ 4\end{array}\right)$

\begin{tabular}{|rr|rr|rrr|}
\hline$U$ & $V$ & $n$ & $m$ & $m_{1}$ & $m_{2}$ & $m_{T}$ \\
\hline-9 & -123 & -20 & -7 & 0 & 2 & 1 \\
-9 & 123 & 21 & -7 & -1 & -1 & 0 \\
-5 & -33 & -5 & -3 & 1 & 0 & 1 \\
-5 & 33 & 6 & -3 & -2 & 1 & 0 \\
-3 & -9 & -1 & -1 & -2 & 0 & 1 \\
-3 & 9 & 2 & -1 & 1 & 1 & 0 \\
-2 & -3 & 0 & 0 & 0 & 1 & 1 \\
-2 & 3 & 1 & 0 & -1 & 0 & 0 \\
-1 & -3 & 0 & 1 & 0 & 0 & 1 \\
-1 & 3 & 1 & 1 & -1 & 1 & 0 \\
\hline
\end{tabular}

\begin{tabular}{|rr|rr|rrr|}
\hline$U$ & $V$ & $n$ & $m$ & $m_{1}$ & $m_{2}$ & $m_{T}$ \\
\hline 0 & -3 & 0 & 2 & -1 & 1 & 1 \\
0 & 3 & 1 & 2 & 0 & 0 & 0 \\
1 & -3 & 0 & 3 & -1 & 0 & 1 \\
1 & 3 & 1 & 3 & 0 & 1 & 0 \\
2 & -9 & -1 & 4 & 1 & 1 & 1 \\
2 & 9 & 2 & 4 & -2 & 0 & 0 \\
4 & -33 & -5 & 6 & -2 & 1 & 1 \\
4 & 33 & 6 & 6 & 1 & 0 & 0 \\
8 & -123 & -20 & 10 & -1 & -1 & 1 \\
8 & 123 & 21 & 10 & 0 & 2 & 0 \\
\hline
\end{tabular}

Theorem B26. The complete set of solutions in rational integers $X, Y$ of the equation

$$
Y^{2}+Y=X^{3}+X^{2}-58 X+1294
$$

is given in Table T26.

TABLE T26. The solutions to (W26) and to $\left(\begin{array}{l}n \\ 2\end{array}\right)=\left(\begin{array}{c}m \\ 6\end{array}\right)$

\begin{tabular}{|rr|ll|rr|}
\hline$X$ & $Y$ & $n$ & $m$ & $m_{1}$ & $m_{2}$ \\
\hline-13 & -5 & & & 0 & -2 \\
-13 & 4 & & & 0 & 2 \\
-7 & -38 & 0 & 2,3 & -1 & 0 \\
-7 & 37 & 1 & 2,3 & 1 & 0 \\
-2 & -38 & 0 & 1,4 & 1 & 1 \\
-2 & 37 & 1 & 1,4 & -1 & -1 \\
2 & -35 & & & -2 & 2 \\
2 & 34 & & & 2 & -2 \\
8 & -38 & 0 & 0,5 & 0 & -1 \\
8 & 37 & 1 & 0,5 & 0 & 1 \\
14 & -59 & & & 2 & 0 \\
14 & 58 & & & -2 & 0 \\
\hline
\end{tabular}

\begin{tabular}{|rr|rr|rr|}
\hline$X$ & $Y$ & $n$ & $m$ & $m_{1}$ & $m_{2}$ \\
\hline 23 & -113 & -1 & $-1,6$ & -1 & 1 \\
23 & 112 & 2 & $-1,6$ & 1 & -1 \\
68 & -563 & -7 & $-3,8$ & 1 & -2 \\
68 & 562 & 8 & $-3,8$ & -1 & 2 \\
133 & -1538 & -20 & $-5,10$ & -2 & 3 \\
133 & 1537 & 21 & $-5,10$ & 2 & -3 \\
233 & -3563 & & & -2 & -1 \\
233 & 3562 & & & 2 & 1 \\
323 & -5813 & -77 & $-9,14$ & 3 & -1 \\
323 & 5812 & 78 & $-9,14$ & -3 & 1 \\
2234 & -105614 & & & 0 & 4 \\
2234 & 105613 & & & 0 & -4 \\
\hline
\end{tabular}

Theorem B28. The complete set of solutions in rational integers $U, V$ of the equation

$$
V^{2}=35 U^{4}-350 U^{3}+945 U^{2}-630 U+11025
$$

is given in Table $\mathrm{T} 28$.

Theorem B34 (Mordell). The complete set of solutions in rational integers $X, Y$ of the equation

$$
Y^{2}+Y=X^{3}-X
$$

is given in Table $\mathrm{T} 34$. 
TABLE T28. The solutions to (Q28) and to $\left(\begin{array}{l}n \\ 2\end{array}\right)=\left(\begin{array}{c}m \\ 8\end{array}\right)$

\begin{tabular}{|rr|rr|rrrrr|}
\hline$U$ & $V$ & $n$ & $m$ & $m_{1}$ & $m_{2}$ & $m_{3}$ & $m_{4}$ & $m_{5}$ \\
\hline-147 & -132195 & & & 1 & 0 & -1 & 0 & -1 \\
-147 & 132195 & & & 0 & 0 & 0 & -1 & 1 \\
-25 & -4445 & & & 1 & 0 & -2 & 0 & 0 \\
-25 & 4445 & & & 0 & 0 & 1 & -1 & 0 \\
-15 & -1785 & & & 1 & -1 & 0 & -1 & 0 \\
-15 & 1785 & & & 0 & 1 & -1 & 0 & 0 \\
-4 & -245 & & & 0 & 1 & 0 & 0 & 0 \\
-4 & 245 & & & 1 & -1 & -1 & -1 & 0 \\
0 & -105 & 0 & 3,4 & 1 & 0 & -1 & -1 & 0 \\
0 & 105 & 1 & 3,4 & 0 & 0 & 0 & 0 & 0 \\
1 & -105 & 0 & 2,5 & 0 & 0 & -1 & 0 & 0 \\
1 & 105 & 1 & 2,5 & 1 & 0 & 0 & -1 & 0 \\
3 & -105 & 0 & 1,6 & 1 & 0 & 0 & 0 & 0 \\
3 & 105 & 1 & 1,6 & 0 & 0 & -1 & -1 & 0 \\
6 & -105 & 0 & 0,7 & 0 & 0 & 0 & -1 & 0 \\
6 & 105 & 1 & 0,7 & 1 & 0 & -1 & 0 & 0 \\
8 & -175 & & & 1 & 1 & -1 & 0 & 0 \\
8 & 175 & & & 0 & -1 & 0 & -1 & 0 \\
10 & -315 & -1 & $-1,8$ & 1 & 0 & 0 & -1 & 1 \\
10 & 315 & 2 & $-1,8$ & 0 & 0 & -1 & 0 & -1 \\
21 & -1995 & -9 & $-3,10$ & 0 & -1 & -1 & -1 & 0 \\
21 & 1995 & 10 & $-3,10$ & 1 & 1 & 0 & 0 & 0 \\
55 & -16275 & -77 & $-7,14$ & 1 & 1 & 0 & 1 & 0 \\
55 & 16275 & 78 & $-7,14$ & 0 & -1 & -1 & -2 & 0 \\
91 & -46305 & -220 & $-10,17$ & 0 & 1 & -1 & -1 & 0 \\
91 & 46305 & 221 & $-10,17$ & 1 & -1 & 0 & 0 & 0 \\
\hline & & & & & & & & \\
\hline
\end{tabular}

TABLE T34. The solutions to (W34) and to $\left(\begin{array}{l}n \\ 3\end{array}\right)=\left(\begin{array}{c}m \\ 4\end{array}\right)$

\begin{tabular}{|rr|rr|r|}
\hline$X$ & $Y$ & $n$ & $m$ & $m_{1}$ \\
\hline-1 & -1 & 0 & 1,2 & 3 \\
-1 & 0 & 0 & 0,3 & -3 \\
0 & -1 & 1 & 1,2 & -1 \\
0 & 0 & 1 & 0,3 & 1 \\
1 & -1 & 2 & 1,2 & -2 \\
\hline
\end{tabular}

\begin{tabular}{|rr|rr|r|}
\hline$X$ & \multicolumn{1}{|c|}{$Y$} & $n$ & $m$ & $m_{1}$ \\
\hline 1 & 0 & 2 & 0,3 & 2 \\
2 & -3 & 3 & & 4 \\
2 & 2 & 3 & $-1,4$ & -4 \\
6 & -15 & 7 & & -6 \\
6 & 14 & 7 & $-4,7$ & 6 \\
\hline
\end{tabular}

TABle T36. The solutions to (C36) and to $\left(\begin{array}{l}n \\ 3\end{array}\right)=\left(\begin{array}{c}m \\ 6\end{array}\right)$

\begin{tabular}{|rr|rr|rrrr|}
\hline$U$ & $V$ & $n$ & $m$ & $m_{1}$ & $m_{2}$ & $m_{3}$ & $m_{4}$ \\
\hline-1 & 0 & 0 & 2,3 & 0 & 1 & 1 & 0 \\
-1 & 1 & 0 & 1,4 & 0 & 0 & 0 & -1 \\
-1 & 3 & 0 & 0,5 & -1 & 0 & 0 & 0 \\
0 & 0 & 1 & 2,3 & 0 & 0 & 0 & 0 \\
0 & 1 & 1 & 1,4 & -1 & 1 & 0 & 0 \\
0 & 3 & 1 & 0,5 & 0 & 0 & 1 & -1 \\
\hline
\end{tabular}

\begin{tabular}{|rr|rr|rrrr|}
\hline$U$ & $V$ & $n$ & $m$ & $m_{1}$ & $m_{2}$ & $m_{3}$ & $m_{4}$ \\
\hline 1 & 0 & 2 & 2,3 & -1 & 0 & 0 & -1 \\
1 & 1 & 2 & 1,4 & 0 & 0 & 1 & 0 \\
1 & 3 & 2 & 0,5 & 0 & 1 & 0 & 0 \\
2 & 6 & 3 & $-1,6$ & -1 & 0 & 1 & -1 \\
8 & 21 & 9 & $-4,9$ & 1 & 0 & 0 & 0 \\
& & & & & & & \\
\hline
\end{tabular}

Theorem B36. The complete set of solutions in rational integers $U, V$ of the equation

$$
15 U^{3}-15 U=V^{3}-4 V^{2}+3 V
$$

is given in Table T36. 
TABLE T46. The solutions to (W46) and to $\left(\begin{array}{l}n \\ 4\end{array}\right)=\left(\begin{array}{c}m \\ 6\end{array}\right)$

\begin{tabular}{|c|c|c|c|c|c|c|}
\hline$X$ & $Y$ & $n$ & $m$ & $m_{1}$ & $m_{2}$ & $m_{3}$ \\
\hline-29 & -32 & & & 1 & 1 & -1 \\
\hline-29 & 31 & & & -1 & -1 & 1 \\
\hline-25 & -88 & & & 0 & 1 & 1 \\
\hline-25 & 87 & & & 0 & -1 & -1 \\
\hline-20 & -113 & 1,2 & 2,3 & 0 & -1 & 0 \\
\hline-20 & 112 & 0,3 & 2,3 & 0 & 1 & 0 \\
\hline-14 & -122 & & & -2 & 0 & 0 \\
\hline-14 & 121 & & & 2 & 0 & 0 \\
\hline-5 & -113 & 1,2 & 1,4 & 1 & 1 & 0 \\
\hline-5 & 112 & 0,3 & 1,4 & -1 & -1 & 0 \\
\hline 5 & -88 & & & -1 & 0 & -1 \\
\hline 5 & 87 & & & 1 & 0 & 1 \\
\hline 14 & -75 & & & -2 & -2 & 0 \\
\hline 14 & 74 & & & 2 & 2 & 0 \\
\hline 16 & -77 & & & 1 & 1 & 1 \\
\hline 16 & 76 & & & -1 & -1 & -1 \\
\hline 20 & -88 & & & 1 & -1 & 0 \\
\hline 20 & 87 & & & -1 & 1 & 0 \\
\hline 25 & -113 & 1,2 & 0,5 & -1 & 0 & 0 \\
\hline 25 & 112 & 0,3 & 0,5 & 1 & 0 & 0 \\
\hline 49 & -320 & & & 0 & 2 & 0 \\
\hline 49 & 319 & & & 0 & -2 & 0 \\
\hline 70 & -563 & & & 0 & 0 & -1 \\
\hline 70 & 562 & $-1,4$ & $-1,6$ & 0 & 0 & 1 \\
\hline 79 & -680 & & & 1 & -1 & 1 \\
\hline 79 & 679 & & & -1 & 1 & -1 \\
\hline 130 & -1463 & & & -1 & 0 & 1 \\
\hline 130 & 1462 & & & 1 & 0 & -1 \\
\hline 250 & -3938 & & & -1 & -2 & 0 \\
\hline 250 & 3937 & & & 1 & 2 & 0 \\
\hline 305 & -5313 & & & 1 & 2 & -1 \\
\hline 305 & 5312 & & & -1 & -2 & 1 \\
\hline 400 & -7988 & & & 2 & 1 & 1 \\
\hline 400 & 7987 & $-7,10$ & $-5,10$ & -2 & -1 & -1 \\
\hline 695 & -18313 & & & -3 & -1 & 0 \\
\hline 695 & 18312 & & & 3 & 1 & 0 \\
\hline 1555 & -61313 & & & 2 & -1 & 0 \\
\hline 1555 & 61312 & & & -2 & 1 & 0 \\
\hline 1645 & -66713 & & & 0 & 2 & 1 \\
\hline 1645 & 66712 & & & 0 & -2 & -1 \\
\hline 18895 & -2597288 & & & -1 & 1 & -2 \\
\hline 18895 & 2597287 & & & 1 & -1 & 2 \\
\hline
\end{tabular}

Theorem B46. The complete set of solutions in rational integers $X, Y$ of the equation

$$
Y^{2}+Y=X^{3}-525 X+10156
$$

is given in Table T46.

Theorem B48. The complete set of solutions in rational integers $U, V$ of the equation

$$
V^{2}=105 U^{4}+210 U^{3}-945 U^{2}-1890 U+11025
$$


TABLE T48. The solutions to (Q48) and to $\left(\begin{array}{l}n \\ 4\end{array}\right)=\left(\begin{array}{c}m \\ 8\end{array}\right)$

\begin{tabular}{|rr|rr|rrr|}
\hline$U$ & $V$ & $n$ & $m$ & $m_{1}$ & $m_{2}$ & $m_{3}$ \\
\hline-3 & -105 & 1,2 & 3,4 & 0 & 1 & 0 \\
-3 & 105 & 0,3 & 3,4 & -1 & 0 & -1 \\
-2 & -105 & 1,2 & 2,5 & 0 & 0 & -1 \\
-2 & 105 & 0,3 & 2,5 & -1 & 1 & 0 \\
0 & -105 & 1,2 & 1,6 & -1 & 1 & -1 \\
0 & 105 & 0,3 & 1,6 & 0 & 0 & 0
\end{tabular} \mid \begin{tabular}{rrrrrrrr|}
$U$ & $V$ & $n$ & $m$ & $m_{1}$ & $m_{2}$ & $m_{3}$ \\
\hline 3 & -105 & 1,2 & 0,7 & -1 & 0 & 0 \\
3 & 105 & 0,3 & 0,7 & 0 & 1 & -1 \\
7 & -525 & & & & 1 & -1 \\
7 & 525 & $-1,4$ & $-1,8$ & -2 & 0 & 0 \\
33 & -11445 & & & -2 & 0 & -1 \\
33 & 11445 & $-9,12$ & $-5,12$ & 1 & 1 & 0 \\
\hline
\end{tabular}

is given in Table T48.

Remarks. Theorem B23 is a bit more general than the result of Avanesov [A], and our proof is rather different.

Theorem B24 is merely a restatement of the main result of [dW1] and [P]. Again the proof we give below is of a different nature.

Theorem B34 is the main result of [M1]. See also J.H. Silverman [Sil1, Exercise 9.13 , p. 275] for a different proof, and see [dW2]. The proof we give below is new.

The meaning of the parameters $m_{1}, \ldots, m_{r}, m_{T}$, given in the Tables T23 to T48, will be made clear in the next sections.

\section{The Cases "Quadratic $=$ CUbiC"}

In this section we shall prove the Theorems B23, B26, B34 and B46, and thus also Theorems A23, A26, A34 and A46. We start by giving in Table 2 some data on the elliptic curves defined by (W23), (W26), (W34) and (W46), namely the minimal discriminant $\Delta$, the $j$-invariant $j$, and the torsion group. These data are easy to compute, e.g. with Apecs.

Further we need the rank $r$, and a basis $P_{1}, \ldots, P_{r}$ for the free part of the Mordell-Weil group. This is more difficult, but can be done. We have the following result.

Proposition 1. The elliptic curves (W23), (W26), (W34) and (W46) have ranks $r$ and bases $P_{1}, \ldots, P_{r}$ for the free parts of their Mordell-Weil groups as in Table 3.

Proof of Proposition 1. We used J.E. Cremona's program mwrank (dated 21 February 1997, see $[\mathrm{Cr}]$ ) on a Sun Sparcstation 4 to compute (unconditionally) the ranks and the Mordell-Weil groups of the four curves. During execution no unusual events occurred.

Briefly, what the program does is this. First a 2-descent is carried out in order to determine a basis for the quotient group $E(\mathbb{Q}) / 2 E(\mathbb{Q})$. What might go wrong but it didn't in these four cases - is that one of the relevant homogeneous spaces to

TABLE 2. Data of elliptic curves

\begin{tabular}{|c|ccc|}
\hline curve & $\Delta$ & $j$ & torsion \\
\hline (W23) & $-130491=-3^{6} \cdot 179$ & $-110592 / 179=-2^{12} \cdot 3^{3} \cdot 179^{-1}$ & trivial \\
(W26) & $-732796875=-3^{5} \cdot 5^{6} \cdot 193$ & $-1404928 / 46899=-2^{12} \cdot 3^{-5} \cdot 7^{3} \cdot 193^{-1}$ & trivial \\
(W34) & 37 & $110592 / 37=2^{12} \cdot 3^{3} \cdot 37^{-1}$ & trivial \\
(W46) & $-35299546875=-3^{7} \cdot 5^{6} \cdot 1033$ & $-1404928 / 3099=-2^{12} \cdot 3^{-1} \cdot 7^{3} \cdot 1033^{-1}$ & trivial \\
\hline
\end{tabular}


TABLE 3. Ranks and bases of Mordell-Weil groups

\begin{tabular}{|c|c|ccc|}
\hline curve & $r$ & $P_{1}$ & $P_{2}$ & $P_{3}$ \\
\hline (W23) & 2 & $(0,4)$ & $(3,4)$ & \\
(W26) & 2 & $(-7,37)$ & $(8,37)$ & \\
(W34) & 1 & $(0,0)$ & & \\
(W46) & 3 & $(25,112)$ & $(-20,112)$ & $(70,562)$ \\
\hline
\end{tabular}

TABLE 4. Relevant constants on heights of basis points

\begin{tabular}{|c|llll|}
\hline curve & (W23) & (W26) & (W34) & (W46) \\
\hline$B$ & 0.3027 & 0.6939 & 0.0256 & 1.561 \\
$H$ & 6.530 & 9.152 & 4.832 & 11.30 \\
runtime & $3 \mathrm{~s}$ & $14 \mathrm{~s}$ & $2 \mathrm{~s}$ & $7 \mathrm{~m} \mathrm{26s}$ \\
\hline
\end{tabular}

be searched for rational points happens to be locally solvable at all primes, without any actual rational point being detected. A successful 2-descent should determine the rank of the curve. Next, an infinite descent has to be done. The purpose of this is to obtain a basis for $E(\mathbb{Q})$, given a basis for $E(\mathbb{Q}) / m E(\mathbb{Q})$ for some $m \geq 2$. Here always $m=2$. To this end usually Zagier's theorem [Sik, Theorem 1.1] is used: if the set

$$
S(B):=\{P \in E(\mathbb{Q}) \mid \hat{h}(P) \leq B\}
$$

contains a complete set of coset representatives for $m E(\mathbb{Q})$ in $E(\mathbb{Q})$, then $S(B)$ generates $E(\mathbb{Q})$. Here $\hat{h}$ is the Néron-Tate height function. For the successful application of Zagier's theorem it is important that $B$ is not too large. The relevant $B$-values for our curves are rather small, see Table 4 .

Finally, the inequality

$$
\frac{1}{2} h(P)-\hat{h}(P) \leq \text { Hdif } \quad \text { for all } P \in E(\mathbb{Q}),
$$

where Hdif is Siksek's [Sik] or Silverman's [Sil2] bound, whichever proves to be smaller, gives an upper bound

$$
h(P) \leq 2 B+2 \text { Hdif }=: H
$$

for the naive height $h(P)$ for all $P \in S(B)$. These bounds, given in Table 4, are not too large, so that a direct search does not cause any problems. We also give runtimes on a Sun Sparcstation 4 in Table 4.

Note that Cremona and Siksek use a canonical height function $\hat{h}$ which is twice the height function used in [ST1]. Also Apecs uses the latter. Here we shall adopt the convention of [ST1].

Further we remark that by a unimodular transformation we arranged the bases such that the least eigenvalue of the Néron-Tate height pairing matrix, called $c_{1}$ below, is as large as possible, see [ST2]. This is of importance for an optimal result of the reduction procedure described below.

To prove Theorems B23, B26, B34 and B46, we use the method of linear forms in elliptic logarithms. We closely follow Stroeker and Tzanakis [ST1], from which paper we also adopt the notation. The proofs are very much a routine matter, taking only a few seconds of runtime on a personal computer. 
TABLE 5. Relevant constants from [ST1]

\begin{tabular}{|c|c|c|c|c|}
\hline curve & (W23) & (W26) & (W34) & (W46) \\
\hline $\begin{array}{r}\left(a_{1}, a_{2}, a_{3}, a_{4}, a_{6}\right) \\
(u, v, w, z)\end{array}$ & $\begin{array}{l}(0,0,1,-9,20) \\
\left(1,0,0,-\frac{1}{2}\right)\end{array}$ & $\begin{array}{l}(0,1,1,-58,1294) \\
\left(1,-\frac{1}{3}, 0,-\frac{1}{2}\right)\end{array}$ & $\begin{array}{l}(0,0,1,-1,0) \\
\left(1,0,0,-\frac{1}{2}\right)\end{array}$ & $\begin{array}{l}(0,0,1,-525,10156) \\
\left(1,0,0,-\frac{1}{2}\right)\end{array}$ \\
\hline$(a, b)$ & $\left(-9, \frac{81}{4}\right)$ & $\left(-\frac{175}{3}, \frac{141875}{108}\right)$ & $\left(-1, \frac{1}{4}\right)$ & $\left(-525, \frac{40625}{4}\right)$ \\
\hline$\gamma$ & $-3.78765 \ldots$ & $-12.7143 \ldots$ & $0.837565 \ldots$ & $-29.4862 \ldots$ \\
\hline$\hat{h}\left(P_{1}\right)$ & $0.170261 \ldots$ & $0.224280 \ldots$ & $0.0255557 \ldots$ & $0.321316 \ldots$ \\
\hline$\hat{h}\left(P_{2}\right)$ & $0.202708 \ldots$ & $0.213613 \ldots$ & & $0.374043 \ldots$ \\
\hline$\hat{h}\left(P_{3}\right)$ & & & & $0.703410 \ldots$ \\
\hline$c_{1}$ & $0.147776 \ldots$ & $0.122596 \ldots$ & $0.0255557 \ldots$ & $0.210864 \ldots$ \\
\hline$c_{2}$ & $7.57530 \ldots$ & $25.4286 \ldots$ & $2.21431 \ldots$ & $58.9725 \ldots$ \\
\hline$X_{0}$ & 8 & 26 & 3 & 59 \\
\hline$c_{3}$ & $0.902545 \ldots$ & $2.21110 \ldots$ & $1.97333 \ldots$ & $2.63185 \ldots$ \\
\hline$\omega$ & $5.89947 \ldots$ & $2.67273 \ldots$ & $5.98691 \ldots$ & $2.06023 \ldots$ \\
\hline$\Im \tau$ & $0.739959 \ldots$ & $0.820738 \ldots$ & $1.22112 \ldots$ & $0.751930 \ldots$ \\
\hline$\phi\left(P_{1}\right)$ & $0.347573 \ldots$ & $0.401475 \ldots$ & $0.189458 \ldots$ & $0.202441 \ldots$ \\
\hline$\phi\left(P_{2}\right)$ & $0.206446 \ldots$ & $0.246042 \ldots$ & & $0.429501 \ldots$ \\
\hline$\phi\left(P_{3}\right)$ & & & & $0.117061 \ldots$ \\
\hline$u_{1}$ & $2.05050 \ldots$ & $1.073036 \ldots$ & $1.13427 \ldots$ & $0.417077 \ldots$ \\
\hline$u_{2}$ & $1.21792 \ldots$ & $0.657605 \ldots$ & & $0.884874 \ldots$ \\
\hline$u_{3}$ & & & & $0.241173 \ldots$ \\
\hline$h_{E}$ & $11.6136 \ldots$ & $14.1554 \ldots$ & $11.6136 \ldots$ & $14.1554 \ldots$ \\
\hline$A_{0}$ & $42.6087 \ldots$ & $36.0874 \ldots$ & $11.6136 \ldots$ & $41.5942 \ldots$ \\
\hline$A_{1}$ & $11.6136 \ldots$ & $14.1554 \ldots$ & $11.6136 \ldots$ & $14.1554 \ldots$ \\
\hline$A_{2}$ & $11.6136 \ldots$ & $14.1554 \ldots$ & & $14.1554 \ldots$ \\
\hline$A_{3}$ & & & & $14.1554 \ldots$ \\
\hline$c_{4}$ & $3.60535 \ldots \times 10^{73}$ & $4.53651 \ldots \times 10^{73}$ & $4.81455 \ldots \times 10^{43}$ & $8.46092 \ldots \times 10^{110}$ \\
\hline $\mathcal{E}$ & $e$ & $e$ & $e$ & $e$ \\
\hline$c_{5}$ & 1 & 1 & 1 & 1 \\
\hline$c_{6}$ & $12.6136 \ldots$ & $15.1554 \ldots$ & $12.6136 \ldots$ & $15.1554 \ldots$ \\
\hline$c_{7}$ & $1.724397 \ldots$ & $1.724397 \ldots$ & 1.0625 & $2.11944 \ldots$ \\
\hline$c_{8}$ & $12.8748 \ldots$ & $15.4167 \ldots$ & $12.6361 \ldots$ & $15.5592 \ldots$ \\
\hline$M_{0}$ & $4.62556 \times 10^{40}$ & $7.50381 \times 10^{40}$ & $2.28469 \times 10^{25}$ & $1.42762 \times 10^{60}$ \\
\hline
\end{tabular}

For an integral point we write $P=m_{1} P_{1}+\ldots+m_{r} P_{r}$, where $P_{1}, \ldots, P_{r}$ is the basis from Table 3 . We write $M=\max _{1 \leq i \leq r}\left|m_{i}\right|$. We omit most of the details of the method, as we do not want to repeat the material of the paper [ST1]. Let us just say that the linear form in elliptic logarithms has the shape

$$
L(P)=m_{0} \omega+m_{1} u_{1}+\ldots+m_{r} u_{r},
$$

where $u_{i}=\omega \phi\left(P_{i}\right)$ are the elliptic logarithms. Here $m_{0} \in \mathbb{Z}$ is taken such that all $\phi$-values are in $[0,1)$. It follows that $\max \left\{M,\left|m_{0}\right|\right\} \leq r M$.

On the one hand we have an upper bound for this linear form:

$$
|L(P)|<4 \sqrt{2} e^{c_{3}-c_{1} M^{2}},
$$

where the constants are defined in [ST1]; their values and other particulars of our equations are given in Table 5. For the calculation of $c_{3}$ the Siksek bound [Sik] was used.

On the other hand the main result by David [D] on linear forms in elliptic logarithms plays an essential rôle, as it provides a lower bound for the linear form:

$$
|L(P)|>\exp \left(-c_{4}\left(\log (M)+c_{7}\right)\left(\log \log (M)+c_{8}\right)^{r+2}\right) .
$$

Together with (2) this yields an absolute upper bound $M_{0}$ for $M$.

The calculations for Table 5 were performed with Apecs 4.2, and required negligible runtime. 
TABle 6. Data of the reduction steps

\begin{tabular}{|c|l|llr|llr|lrr|}
\hline curve & $M_{0}$ & $C$ & $d>$ & $M_{1}$ & $C$ & $d>$ & $M_{2}$ & $C$ & $d>$ & $M_{3}$ \\
\hline (W23) & $4.62556 \times 10^{40}$ & $10^{123}$ & $1.62319 \times 10^{41}$ & 36 & $10^{6}$ & 121.136 & 9 & $10^{4}$ & 31.8904 & 7 \\
(W26) & $7.50381 \times 10^{40}$ & $10^{124}$ & $2.22699 \times 10^{41}$ & 39 & $10^{6}$ & 138.701 & 10 & $10^{5}$ & 52.9622 & 9 \\
(W34) & $2.28469 \times 10^{25}$ & $10^{51}$ & $7.58375 \times 10^{25}$ & 49 & $10^{4}$ & 110.788 & 18 & $10^{3}$ & 61.4003 & 16 \\
(W46) & $1.42762 \times 10^{60}$ & $10^{243}$ & $5.88376 \times 10^{60}$ & 44 & $10^{9}$ & 164.322 & 10 & $10^{7}$ & 36.9323 & 9 \\
\hline
\end{tabular}

To reduce the large upper bound $M_{0}$, we apply lattice base reduction to the lattice spanned by the columns of the matrix

$$
\mathcal{A}=\left(\begin{array}{ccccc}
1 & 0 & \ldots & 0 & 0 \\
0 & 1 & \ldots & 0 & 0 \\
\vdots & \vdots & \ddots & \vdots & \vdots \\
0 & 0 & \ldots & 1 & 0 \\
{\left[C u_{1}\right]} & {\left[C u_{2}\right]} & \ldots & {\left[C u_{r}\right]} & {[C \omega]}
\end{array}\right)
$$

where $C$ is a large constant, of the size of $M_{0}^{r+1}$, and where [·] denotes rounding to the nearest integer. For a possible solution of our elliptic equation we look at the lattice point

$$
\mathcal{A}\left(m_{1}, \ldots, m_{r}, m_{0}\right)^{\top}=\left(m_{1}, \ldots, m_{r}, \lambda\right)^{\top},
$$

where $\lambda$ thus is a good approximation to $C L(P)$, viz.

$$
|\lambda-C L(P)| \leq \frac{1}{2}\left(r M_{0}+\left|m_{0}\right|\right) \leq r M_{0} .
$$

We applied Zagier's algorithm for computing the values of $\phi\left(P_{i}\right)$ to the desired precision (of somewhat more than $(r+1) \log _{10} M_{0}$ decimal digits). For each of the four lattices we computed a reduced basis by the LLL-algorithm. These computations were done by Pari 1.39 .

From the reduced basis we find a lower bound $d$ for the length of the shortest nonzero lattice vector. We may assume that $d$ is large enough. If it isn't, then we have to try a larger value of $C$. We find that either $m_{1}=\ldots=m_{r}=0$, or

$$
|\lambda| \geq \sqrt{d^{2}-m_{1}^{2}-\ldots-m_{r}^{2}} \geq \sqrt{d^{2}-r M_{0}^{2}},
$$

and thus

$$
|L(P)| \geq \frac{1}{C}\left(\sqrt{d^{2}-r M_{0}^{2}}-r M_{0}\right) .
$$

Together with inequality (2) this yields a reduced upper bound $M_{1}$ for $M$, namely

$$
M_{1}=\left\lfloor\sqrt{\frac{1}{c_{1}}\left(\log (4 \sqrt{2} C)+c_{3}-\log \left(\sqrt{d^{2}-r M_{0}^{2}}-r M_{0}\right)\right)}\right\rfloor .
$$

Iterating the procedure, we reduced $M_{1}$ further to $M_{2}$ and finally to $M_{3}$. In Table 6 we list the values for $C$ that we chose, the values for $d$ that follow from the application of the LLL-algorithm, and the reduced upper bounds $M_{1}, M_{2}, M_{3}$ for $M$.

We checked all points $P$ corresponding to $r$-tuples $\left(m_{1}, \ldots, m_{r}\right)$ with $M \leq M_{3}$ for being solutions to inequality (2). The solutions thus found we checked for integrality of the coordinates $X, Y$ of $P$. These computations we again did in 
TABle 7. Runtimes

\begin{tabular}{|c|lll|}
\hline curve & comput. of $\phi\left(P_{i}\right)$ & reduction & small solut. \\
\hline (W23) & $3 \mathrm{~s}$ & $<1 \mathrm{~s}$ & $6 \mathrm{~s}$ \\
(W26) & $3 \mathrm{~s}$ & $<1 \mathrm{~s}$ & $10 \mathrm{~s}$ \\
(W34) & $<1 \mathrm{~s}$ & $<1 \mathrm{~s}$ & $1 \mathrm{~s}$ \\
(W46) & $25 \mathrm{~s}$ & $8 \mathrm{~s}$ & $4 \mathrm{~m} 28 \mathrm{~s}$ \\
\hline
\end{tabular}

Apecs. This produced the results mentioned above, and thus completes our proof. Finally we give in Table 7 the runtimes on a Pentium $75 \mathrm{Mhz}$ personal computer.

\section{The Cases "Quadratic = Quartic"}

In this section we prove Theorems B24, B28 and B48, and thus also Theorems A24, A28 and A48. We use the method of linear forms in elliptic logarithms. We follow Tzanakis $[\mathrm{T}]$, but at certain points use slight variations in the arguments. The proofs are to some extent a routine matter, but now things are essentially more complicated than in the previous section. We start by giving some information on the elliptic curves represented by the equations (Q24), (Q28) and (Q48).

The birational transformations

$$
\left\{\begin{array} { l } 
{ U = \frac { 6 X _ { 1 } - 1 8 } { X _ { 1 } + Y _ { 1 } - 1 7 } , } \\
{ V = \frac { - 2 7 X _ { 1 } ^ { 2 } + 3 Y _ { 1 } ^ { 2 } + 8 8 2 X _ { 1 } + 8 4 Y _ { 1 } - 4 1 6 7 } { ( X _ { 1 } + Y _ { 1 } - 1 7 ) ^ { 2 } } , }
\end{array} \quad \left\{\begin{array}{l}
X_{1}=\frac{-U^{2}-6 U+6 V+18}{U^{2}} \\
Y_{1}=\frac{18 U^{3}-18 U^{2}-6 U V-54 U+36 V+108}{U^{3}}
\end{array}\right.\right.
$$

relate equation (Q24) and the minimal model

$$
Y_{1}^{2}=X_{1}^{3}-147 X_{1}+610
$$

The birational transformations

$$
\begin{aligned}
& \left\{\begin{array}{l}
U=\frac{210 X_{1}+130410}{3 X_{1}+Y_{1}+35805} \\
V=\frac{195615 X_{1}^{2}+105 Y_{1}^{2}+340341750 X_{1}-7127820 Y_{1}-226983637125}{\left(3 X_{1}+Y_{1}+35805\right)^{2}},
\end{array}\right. \\
& \left\{\begin{array}{l}
X_{1}=\frac{315 U^{2}-630 U+210 V+22050}{U^{2}} \\
Y_{1}=\frac{-36750 U^{3}+198450 U^{2}-630 U V-198450 U+44100 V+4630500}{U^{3}}
\end{array}\right.
\end{aligned}
$$

relate equation (Q28) and the minimal model

$$
Y_{1}^{2}=X_{1}^{3}-1620675 X_{1}+385103250
$$


TABle 8. Data of elliptic curves

\begin{tabular}{|c|ccc|}
\hline curve & $\Delta$ & $j$ & torsion \\
\hline (W24) & $42550272=$ & $470596 / 57=$ & $\mathbb{Z} / 2 \mathbb{Z}=\langle T\rangle$, \\
& $2^{10} \cdot 3^{7} \cdot 19$ & $2^{2} \cdot 3^{-1} \cdot 7^{6} \cdot 19^{-1}$ & $T=(5,0)$ \\
\hline (W28) & $208370506291920000000=$ & $114354828 / 50615=$ & trivial \\
& $2^{10} \cdot 3^{7} \cdot 5^{7} \cdot 7^{6} \cdot 53 \cdot 191$ & $2^{2} \cdot 3^{5} \cdot 5^{-1} \cdot 7^{6} \cdot 53^{-1} \cdot 191^{-1}$ & \\
\hline (W48) & $999362923039687500=$ & $112678587 / 27620=$ & trivial \\
& $2^{2} \cdot 3^{9} \cdot 5^{7} \cdot 7^{6} \cdot 1381$ & $2^{-2} \cdot 3^{3} \cdot 5^{-1} \cdot 7^{3} \cdot 23^{3} \cdot 1381^{-1}$ & \\
\hline
\end{tabular}

TABLE 9. Ranks and bases of Mordell-Weil groups

\begin{tabular}{|c|c|ccccc|}
\hline curve & $r$ & $P_{1}$ & $P_{2}$ & $P_{3}$ & $P_{4}$ & $P_{5}$ \\
\hline (W24) & 2 & $(11,18)$ & $(29,144)$ & & & \\
(W28) & 5 & $(105,14700)$ & $(-1365,7350)$ & $(-315,-29400)$ & $(210,7350)$ & $\left(-\frac{4235}{9},-\frac{872200}{27}\right)$ \\
(W48) & 3 & $(-236,11143)$ & $(79,5473)$ & $\left(\frac{631}{4},-\frac{22681}{8}\right)$ & & \\
\hline
\end{tabular}

The birational transformations

$$
\begin{aligned}
& \left\{\begin{array}{l}
U=\frac{105 X_{1}-18690}{5 X_{1}+Y_{1}-2403}, \\
V=\frac{-55965 X_{1}^{2}+105 X_{1} Y_{1}+105 Y_{1}^{2}+70087815 X_{1}+336420 Y_{1}-11480080185}{\left(5 X_{1}+Y_{1}-2403\right)^{2}},
\end{array}\right. \\
& \left\{\begin{array}{l}
X_{1}=\frac{-157 U^{2}-945 U+105 V+11025}{2 U^{2}} \\
Y_{1}=\frac{5591 U^{3}-49140 U^{2}-525 U V-154350 U+11025 V+1157625}{2 U^{3}}
\end{array}\right.
\end{aligned}
$$

relate equation $(\mathrm{Q} 48)$ and the minimal model

$$
Y_{1}^{2}+X_{1} Y_{1}=X_{1}^{3}-X_{1}^{2}-332817 X_{1}+56191841 .
$$

In Table 8 some data on these elliptic curves are given, namely the minimal discriminant $\Delta$, the $j$-invariant $j$, and the torsion group. In the case of nontrivial torsion generators of the torsion group are given.

Further we need the rank $r$, and a basis $P_{1}, \ldots, P_{r}$ for the free part of the Mordell-Weil group. We have the following result. In the sequel coordinates of points on the elliptic curves are given for the minimal models (W24), (W28) and (W48), unless explicitly stated otherwise.

Proposition 2. The elliptic curves (W24), (W28) and (W48) have ranks $r$ and bases $P_{1}, \ldots, P_{r}$ for the free parts of their Mordell-Weil groups as in Table 9.

Proof of Proposition 2. Again we use Cremona's mwrank. No difficulties were encountered with (W24). But with (W28) and (W48) mwrank ran into trouble, as the upper bounds $H$ the program computed for these curves turned out to be too large. Table 10 gives the relevant values found by mwrank, where the runtimes on a Sun Sparcstation 4 for (W28) and (W48) reflect the time it took mrank (without 'w') to compute a set of representatives for $E(\mathbb{Q}) / 2 E(\mathbb{Q})$.

Starting from here, we calculated by hand improved sets of representatives resulting in much better values for $B$. The bounds $H$ on the logarithmic height so obtained appear to be small enough for Cremona's findinf to run successfully. This is true for (W48), but in the case of (W28) findinf produces erroneous results like points not on the curve, or it overlooks existing relations between points. John 
TABLE 10. Relevant constants on heights of basis points

\begin{tabular}{|c|lll|}
\hline curve & (W24) & $(\mathrm{W} 28)$ & $(\mathrm{W} 48)$ \\
\hline$B$ & 0.8105 & 5.117 & 2.734 \\
$H$ & 9.063 & 22.79 & 17.93 \\
runtime & $13 \mathrm{~s}$ & $6 \mathrm{~h} 54 \mathrm{~m}$ & $75 \mathrm{~h} 9 \mathrm{~m}$ \\
\hline
\end{tabular}

TABLE 11. Relevant constants on heights of basis points

\begin{tabular}{|c|ll|}
\hline curve & $(\mathrm{W} 28)$ & $(\mathrm{W} 48)$ \\
\hline$B$ & 3.199 & 1.711 \\
$H$ & 16.265 & 12.634 \\
runtime (findinf) & $\approx 2$ weeks & $2 \mathrm{~h}$ \\
\hline
\end{tabular}

Cremona told us that these errors are most likely due to roundoff. We give the improved values for $B$ and $H$ in Table 11 . Here the runtime given in the case of (W28) is the expected total runtime for a hypothetically completely succesful run of the program findinf, estimated from the amount of work it had completed when it ran into serious trouble.

In the case of (W28) we use the sieving technique described in [Sik, section 4.1]. As a result of the 2 -descent, we know that the points $P_{i}(i=1, \ldots, 5)$ of Table 9 generate a subgroup of odd index $m$ in $E(\mathbb{Q})$.

First we have to find an upper bound for $m$. This we do by [Sik, Theorem 3.1]. To compute $\lambda$ such that $\hat{h}(Q) \leq \lambda$ has no solutions $Q \in E(\mathbb{Q})$ other than the point at infinity, we try to show that there are no such points with $\hat{h}(Q)<\hat{h}\left(P_{3}\right)=$ $0.794302 \ldots$ Because Siksek's upper bound for $\frac{1}{2} h(Q)-\hat{h}(Q)$ is Hdif $=4.93332 \ldots$, we search, using Cremona's findinf, for the points with $h(Q)<2\left(\hat{h}\left(P_{3}\right)+\right.$ Hdif $)<$ 11.4553. This search took 13 minutes and 40 seconds on a Sun Sparcstation 4 , and revealed that there are no points $Q$ with $\hat{h}(Q) \leq \lambda=0.794302$.

The regulator of $P_{1}, \ldots, P_{5}$ is $R=28.3648 \ldots$ Now [Sik, Theorem 3.1] tells us that

$$
m \leq\left(\frac{8 R}{(2 \lambda)^{5}}\right)^{1 / 2}=4.73582 \ldots,
$$

so that $m \leq 3$. Note that Siksek uses twice our height.

Now we start the sieving, to show that $m \neq 3$. Let

$$
V_{3}=\left\{\left(a_{1}, \ldots, a_{5}\right) \mid a_{i} \in\{-1,0,1\}, a_{1} P_{1}+\ldots+a_{5} P_{5} \in 3 E(\mathbb{Q})\right\} .
$$

As $m=3$ implies the existence of a nonzero element of $V_{3}$, we intend to show that $V_{3}=\{(0,0,0,0,0)\}$.

Let $v$ be a prime of good reduction, such that $\# E\left(\mathbb{F}_{v}\right)$ is divisible by 3 , but not by 9. Put $\ell=\frac{1}{3} \# E\left(\mathbb{F}_{v}\right)$. Then the group $\ell E\left(\mathbb{F}_{v}\right)$ has order 3. Compute a generator $G$ of this group, and compute $m_{1}, \ldots, m_{5} \in\{-1,0,1\}$ such that $\ell P_{i} \equiv m_{i} G(\bmod v)$. Now, if $\left(a_{1}, \ldots, a_{5}\right) \in V_{3}$, then $\ell\left(a_{1} P_{1}+\ldots+a_{5} P_{5}\right) \in 3 \ell E(\mathbb{Q})$, so that

$$
\left(m_{1} a_{1}+\ldots+m_{5} a_{5}\right) G \equiv a_{1} \ell P_{1}+\ldots+a_{5} \ell P_{5} \equiv 0(\bmod v) \text {. }
$$


TABLE 12. Relations found in Siksek sieving

\begin{tabular}{|r|r|r|rrrrr|}
\hline$v$ & $\ell$ & $G$ & $m_{1}$ & $m_{2}$ & $m_{3}$ & $m_{4}$ & $m_{5}$ \\
\hline 11 & 5 & $5 P_{1}$ & 1 & 0 & 1 & 1 & 1 \\
17 & 7 & $7 P_{1}$ & 1 & 0 & 1 & -1 & -1 \\
23 & 10 & $10 P_{1}$ & 1 & -1 & -1 & 1 & -1 \\
31 & 11 & $11 P_{1}$ & 1 & 1 & 0 & -1 & 1 \\
43 & 16 & $16 P_{2}$ & 0 & 1 & -1 & 1 & -1 \\
47 & 20 & $20 P_{2}$ & 0 & 1 & -1 & -1 & 0 \\
59 & 20 & $20 P_{1}$ & 1 & 1 & 1 & 0 & 1 \\
\hline
\end{tabular}

As $G$ generates a group of order 3 in $E\left(\mathbb{F}_{v}\right)$, we find that

$$
m_{1} a_{1}+\ldots+m_{5} a_{5} \equiv 0(\bmod 3)
$$

which constitutes a relation on the $a_{i}$.

We do the above computation for a number of primes $v$, to find as many independent relations as needed or as possible. It turned out that seven primes, starting from the smallest, is a sufficient number in our situation to find 5 independent relations, thus proving that $V_{3}=\{(0,0,0,0,0)\}$. In Table 12 we give the primes $v$, and for each prime the number $\ell$, the generator $G$, and the relation $m_{1}, \ldots, m_{5}$. Note that the $7 \times 5$ matrix of the $m_{i}$, defined $(\bmod 3)$, has rank 5 indeed.

Again note that each Mordell-Weil basis given is optimal for the least eigenvalue of the Néron-Tate height pairing matrix.

We note that there is some symmetry, namely the irrelevance of the sign of $V$, that we now describe. We take the point $Q$ as follows:

$$
\begin{array}{ll}
\text { (W24): } & Q=-P_{1}+P_{2}+T=(3,-14) \\
\text { (W28): } & Q=P_{1}-P_{3}-P_{4}=(-621,33942) \\
\text { (W48): } & Q=-P_{1}+P_{2}-P_{3}=(178,-1691)
\end{array}
$$

If a point $P$ on the curve has coordinates $U(P), V(P)$ on the quartic model, then we have

$$
\left\{\begin{array}{l}
U(-P+Q)=U(P) \\
V(-P+Q)=-V(P) .
\end{array}\right.
$$

As $\Delta>0$, the Weierstrass curve $E(\mathbb{R})$ has a compact component and an infinite component. We denote the infinite component by $E_{0}(\mathbb{R})$. Since $Q$ is on the compact component, it follows that of the two points $P,-P+Q$ always one is on $E_{0}(\mathbb{R})$. Thus from now on we may assume without loss of generality that our point $P$ is on the infinite component. In the case of (W24) this implies that $P$ is in the free part of the Mordell-Weil group, i.e. that we can forget about the torsion point.

In the case of equation (W24) there is yet another symmetry:

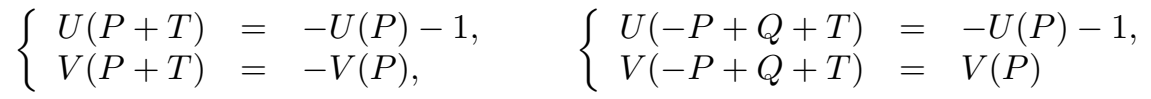

(note that $Q+T$ is in the free part of the Mordell-Weil group). It follows that in this case we may as well assume without loss of generality that $U \geq 0$.

In Table 14 (later) we give the relevant constants from [T].

For all three of our elliptic curves we have that $x_{0}>e_{1}$, so that $U>U_{0}$ implies that the point $P$ is on $E_{0}(\mathbb{R})$. The group $E_{0}(\mathbb{Q})$ of rational points on this infinite 
TABLE 13. Bases of relevant subgroups of Mordell-Weil groups

\begin{tabular}{|c|ccccc|}
\hline curve & $R_{1}$ & $R_{2}$ & $R_{3}$ & $R_{4}$ & $R_{5}$ \\
\hline (W24) & $P_{1}=(11,18)$ & $P_{2}=(29,144)$ & & & $-P_{4}+P_{5}=$ \\
\hline (W28) & $P_{1}+P_{4}=$ & $P_{2}+P_{4}=$ & $P_{3}+P_{4}=$ & $P_{1}+P_{2}+P_{3}+P_{4}=$ & $(1605,-43800)$ \\
& $(4585,298900)$ & $(1155,-7350)$ & $(5005,-343000)$ & $\left(\frac{10990}{9}, \frac{406700}{27}\right)$ & $(12=$ \\
\hline (W48) & $P_{1}-P_{2}=$ & $P_{1}+P_{2}=$ & $P_{2}-P_{3}=$ & & \\
& $(2914,152893)$ & $(464,993)$ & $(989,25843)$ & & \\
\hline
\end{tabular}

component is a subgroup of $E(\mathbb{Q})$ of index 2. Clearly all that we need is a basis $R_{1}, \ldots, R_{r}$ for this smaller group only. It's easy to show that we can take this basis as in Table 13. Note that we took these bases such that an optimal least eigenvalue of the Néron-Tate height pairing matrix is obtained.

For the rational point $P$ we now put

$$
P=m_{1} P_{1}+\ldots+m_{r} P_{r}=m_{1}^{\prime} R_{1}+\ldots+m_{r}^{\prime} R_{r} .
$$

For the solutions we give in Tables T24, T28 and T48 the corresponding values of $m_{1}, \ldots m_{r}$, also for the points not on the infinite component $E_{0}(\mathbb{R})$. In the case of (Q24) we have $P=m_{T} T+m_{1} P_{1}+m_{2} P_{2}$, with $m_{T}=0$ if $P$ is on $E_{0}(\mathbb{R})$, and $m_{T}=1$ if $P$ is in the compact component. But as argued above we may assume that $P$ is on $E_{0}(\mathbb{R})$, i.e. $m_{T}=0$. Note that

$$
\begin{array}{ll}
\text { for }(\mathrm{Q} 24): & m_{1}^{\prime}=m_{1}, m_{2}^{\prime}=m_{2}, \\
\text { for }(\mathrm{Q} 28): & m_{1}^{\prime}=\frac{1}{2}\left(m_{1}-m_{2}-m_{3}+m_{4}+m_{5}\right), \\
& m_{2}^{\prime}=\frac{1}{2}\left(-m_{1}+m_{2}-m_{3}+m_{4}+m_{5}\right), \\
& m_{3}^{\prime}=\frac{1}{2}\left(-m_{1}-m_{2}+m_{3}+m_{4}+m_{5}\right), \\
& m_{4}^{\prime}=\frac{1}{2}\left(m_{1}+m_{2}+m_{3}-m_{4}-m_{5}\right), m_{5}^{\prime}=m_{5}, \\
\text { for (Q48): } & m_{1}^{\prime}=\frac{1}{2}\left(m_{1}-m_{2}-m_{3}\right), \\
& m_{2}^{\prime}=\frac{1}{2}\left(m_{1}+m_{2}+m_{3}\right), m_{3}^{\prime}=-m_{3} .
\end{array}
$$

In any case we put

$$
M=\max \left\{\left|m_{1}^{\prime}\right|, \ldots,\left|m_{r}^{\prime}\right|\right\},
$$

and we have

$$
\phi(P)=m_{0}^{\prime}+m_{1}^{\prime} \phi\left(R_{1}\right)+\ldots+m_{r}^{\prime} \phi\left(R_{r}\right),
$$

where we take $m_{0}^{\prime} \in \mathbb{Z}$ such that $0 \leq \phi(P)<1$. It follows that $\left|m_{0}^{\prime}\right| \leq r M$. An interesting point to notice about (Q24) is that $P_{0}$ is not independent of $R_{1}$ and $R_{2}$. Indeed, we have $2 P_{0}=-R_{1}+R_{2}$. In terms of $\phi$ we have

$$
2 \phi\left(P_{0}\right)=-\phi\left(R_{1}\right)+\phi\left(R_{2}\right)+1 .
$$

It follows that we do not have to count with $P_{0}$ anymore, when we multiply the linear form by 2 and adapt $m_{i}^{\prime}$. Equally interesting is the fact that in both cases $(\mathrm{Q} 28)$ and $(\mathrm{Q} 48)$ a similar relation (over $\mathbb{Q}$ ) does not exist, or so it seems, at least not with very small coefficients.

We put $u_{i}=\omega \phi\left(R_{i}\right)$ for $i=1, \ldots, r$, and $u_{0}=\omega \phi\left(P_{0}\right)$. Our linear form in elliptic logarithms then is

$$
L(P)=m_{0}^{\prime} \omega+m_{1}^{\prime} u_{1}+\ldots+m_{r}^{\prime} u_{r}+u_{0},
$$


for which we have the inequality

$$
|L(P)|<c_{9} e^{\frac{1}{2} c_{10}+c_{11}-c_{1} M^{2}}
$$

On the other hand, in the cases of (Q28) and (Q48), David [D] gives

$$
|L(P)|>\exp \left(-c_{4}\left(\log (r M)+c_{5}\right)\left(\log \log (r M)+c_{6}\right)^{r+3}\right) .
$$

But in the case of (Q24) we use

$$
2 L(P)=\left(2 m_{0}^{\prime}+1\right)+\left(2 m_{1}^{\prime}-1\right) u_{1}+\left(2 m_{2}^{\prime}+1\right) u_{2}
$$

instead of $L(P)$ itself, as it has one term less. Then, based on $r=2$ and the inequality $\max \left\{\left|2 m_{0}^{\prime}+1\right|,\left|2 m_{1}^{\prime}-1\right|,\left|2 m_{2}^{\prime}+1\right|\right\} \leq 4 M+1$, David [D] gives

$$
|2 L(P)|>\exp \left(-c_{4}\left(\log (4 M+1)+c_{5}\right)\left(\log \log (4 M+1)+c_{6}\right)^{4}\right) .
$$

Whatever the case may be, together with (3) this yields an absolute upper bound $M_{0}$ for $M$.

In Table 14 we take $A_{0}$ corresponding to $\omega$, and $A_{0}^{\prime}$ corresponding to $u_{0}$. To compute $A_{0}^{\prime}$ we had to estimate the Néron-Tate height of the non-rational point $P_{0}$. We used $\hat{h}(P) \leq c_{11}+\frac{1}{2} h\left(X_{1}(P)\right)$, where $h(X)$ is the absolute logarithmic Weil height for the algebraic number $X$. For computing $c_{11}$ we always used the minimal model. Note that $D \leq 2$, since the coordinates of $P_{0}$ are quadratic.

The reduction in the case of (Q24) goes just as in the previous section, based on inequality (3), but working with $2 L(P)$. We take

$$
\mathcal{A}=\left(\begin{array}{ccc}
1 & 0 & 0 \\
0 & 1 & 0 \\
{\left[C u_{1}\right]} & {\left[C u_{2}\right]} & {[C \omega]}
\end{array}\right),
$$

and we look at the lattice point

$$
\mathcal{A}\left(2 m_{1}^{\prime}-1,2 m_{2}^{\prime}+1,2 m_{0}^{\prime}+1\right)^{\top}=\left(2 m_{1}^{\prime}-1,2 m_{2}^{\prime}+1, \lambda\right)^{\top},
$$

where $\lambda$ thus is a good approximation to $2 C L(P)$, viz.

$$
|\lambda-2 C L(P)| \leq \frac{1}{2}\left(2\left(2 M_{0}+1\right)+2\left|m_{0}^{\prime}\right|+1\right) \leq 4 M_{0}+\frac{3}{2},
$$

because $\left|m_{0}\right| \leq 2 M_{0}$. As in the previous section we obtain by (3) a reduced upper bound $M_{1}$ for $M$, namely

$$
M_{1}=\left\lfloor\sqrt{\frac{1}{c_{1}}\left(\log \left(2 c_{9} C\right)+\frac{1}{2} c_{10}+c_{11}-\log \left(\sqrt{d^{2}-2\left(2 M_{0}+1\right)^{2}}-\left(4 M_{0}+\frac{3}{2}\right)\right)\right)}\right\rfloor .
$$

In Table 15 we give the data for this reduction.

In the cases (Q28) and (Q48) the reduction procedure is slightly different, because now the linear forms in elliptic logarithms are inhomogeneous. We take the lattice as usual, spanned by the columns of

$$
\mathcal{A}=\left(\begin{array}{ccccc}
1 & 0 & \ldots & 0 & 0 \\
0 & 1 & \ldots & 0 & 0 \\
\vdots & \vdots & \ddots & \vdots & \vdots \\
0 & 0 & \ldots & 1 & 0 \\
{\left[C u_{1}\right]} & {\left[C u_{2}\right]} & \ldots & {\left[C u_{r}\right]} & {[C \omega]}
\end{array}\right)
$$

and look at the point

$$
\mathbf{y}=\left(0,0, \ldots, 0,-\left[C u_{0}\right]\right)^{\top},
$$


TABLE 14. Relevant constants from [T]

\begin{tabular}{|c|c|c|c|}
\hline curve & (Q24) & (Q28) & $(\mathrm{Q} 48)$ \\
\hline $\begin{array}{r}(a, b, c) \\
d, e)\end{array}$ & $\begin{array}{c}(3,6,-3) \\
-6,3)\end{array}$ & $\begin{array}{c}(35,-350,945 \\
-630.105)\end{array}$ & $\begin{array}{c}(105,210,-945 \\
-1890.105)\end{array}$ \\
\hline$\left(a_{1}, a_{2}, a_{3}\right)$ & $(-2,-4,36$ & $(-6,936,-73500$ & $(-18,-1026,44100$ \\
\hline$a_{4}$ & -108 & -1543500 & -4630500 \\
\hline$\left.a_{6}\right)$ & 432) & $-1444716000)$ & $4750893000)$ \\
\hline$(A, B)$ & $(-147,610)$ & $\begin{array}{l}(-1620675 \\
385103250)\end{array}$ & $\begin{array}{l}(-5325075, \\
3590952750)\end{array}$ \\
\hline$\left(X_{1}, Y_{1}\right)$ & $(x, y)$ & $(x, y)$ & $\left(\frac{1}{4}(x+1)\right.$ \\
\hline & & & $\left.\frac{1}{8}(-x+y-1)\right)$ \\
\hline$\sigma$ & 1 & -1 & 1 \\
\hline$x_{0}$ & $-1+6 \sqrt{3}$ & $315+210 \sqrt{35}$ & $-315+210 \sqrt{105}$ \\
\hline$u_{0}$ & 0 & 9.38860 & 0 \\
\hline$e_{1}$ & $8.82475 \ldots$ & $1131.51 \ldots$ & $1835.35 \ldots$ \\
\hline$e_{2}$ & 5 & $246.906 \ldots$ & $755.246 \ldots$ \\
\hline$e_{3}$ & $-13.8247 \ldots$ & $-1378.42 \ldots$ & $-2590.60 \ldots$ \\
\hline$\omega$ & $1.96209 \ldots$ & $0.160046 \ldots$ & $0.130311 \ldots$ \\
\hline$\Im \tau$ & $1.41977 \ldots$ & $1.14814 \ldots$ & $1.28813 \ldots$ \\
\hline$U_{0}$ & 0 & 9.38860 & \\
\hline$P_{0}$ & $\begin{array}{c}(-1+6 \sqrt{3} \\
18-6 \sqrt{3})\end{array}$ & $\begin{array}{l}(315+210 \sqrt{35} \\
36750+630 \sqrt{35})\end{array}$ & $\left(\frac{-157+105 \sqrt{105}}{2}\right.$, \\
\hline$\hat{h}\left(R_{1}\right)$ & $0.202321 \ldots$ & $1.76131 \ldots$ & $1.12812^{2} \ldots$ \\
\hline$\hat{h}\left(R_{2}\right)$ & $0.506291 \ldots$ & $1.17118 \ldots$ & $1.00476 \ldots$ \\
\hline$\hat{h}\left(R_{3}\right)$ & & $2.00293 \ldots$ & $1.52711 \ldots$ \\
\hline$\hat{h}\left(R_{4}\right)$ & & $2.80407 \ldots$ & \\
\hline$\hat{h}\left(R_{5}\right)$ & & $2.10666 \ldots$ & \\
\hline$c_{1}$ & $0.194012 \ldots$ & $0.612991 \ldots$ & $0.613916 \ldots$ \\
\hline$\phi\left(R_{1}\right)$ & $0.352986 \ldots$ & $0.185963 \ldots$ & $0.142707 \ldots$ \\
\hline$\phi\left(R_{2}\right)$ & $0.192459 \ldots$ & $0.540398 \ldots$ & $0.469004 \ldots$ \\
\hline$\phi\left(R_{3}\right)$ & & $0.822229 \ldots$ & $0.252395 \ldots$ \\
\hline$\phi\left(R_{4}\right)$ & & $0.422353 \ldots$ & \\
\hline$\phi\left(R_{5}\right)$ & & $0.664566 \ldots$ & \\
\hline$\phi\left(P_{0}\right)$ & & $0.342498 \ldots$ & $0.491395 \ldots$ \\
\hline$c_{9}$ & $\frac{1}{3} \sqrt{3}=0.577350 \ldots$ & $0.234326 \ldots$ & 0.104791 \\
\hline$c_{10}$ & $\log (10)=2.30258 \ldots$ & 7.73976 & 7.71756 \\
\hline$c_{11}$ & $3.28540 \ldots$ & $5.61238 \ldots$ & $4.87006 \ldots$ \\
\hline$\hat{h}\left(P_{0}\right) \leq$ & & 9.15817 & 8.00858 \\
\hline$\overline{u_{1}}$ & $0.692592 \ldots$ & $0.0297628 \ldots$ & $0.0185963 \ldots$ \\
\hline$u_{2}$ & $0.377623 \ldots$ & $0.0864891 \ldots$ & $0.0611166 \ldots$ \\
\hline$u_{3}$ & & $0.131595 \ldots$ & $0.0328899 \ldots$ \\
\hline$u_{4}$ & & $0.0675963 \ldots$ & \\
\hline$u_{5}$ & & $0.106361 \ldots$ & \\
\hline$u_{0}$ & & $0.0548158 \ldots$ & $0.0640343 \ldots$ \\
\hline$h_{E}$ & $13.0617 \ldots$ & $19.0758 \ldots$ & $21.3085 \ldots$ \\
\hline$D$ & 1 & 2 & 2 \\
\hline$A_{0}$ & $13.3810 \ldots$ & $19.0758 \ldots$ & $21.3085 \ldots$ \\
\hline$A_{1}$ & $13.0617 \ldots$ & $19.0758 \ldots$ & $21.3085 \ldots$ \\
\hline$A_{2}$ & $13.0617 \ldots$ & $19.0758 \ldots$ & $21.3085 \ldots$ \\
\hline$A_{3}$ & & $19.0758 \ldots$ & $21.3085 \ldots$ \\
\hline$A_{4}$ & & $19.0758 \ldots$ & \\
\hline$A_{5}$ & & $19.0758 \ldots$ & \\
\hline$A_{0}^{\prime}$ & & $19.0758 \ldots$ & $21.3085 \ldots$ \\
\hline$c_{4}$ & $1.43221 \times 10^{73}$ & $5.61880 \times 10^{277}$ & $2.07088 \times 10^{160}$ \\
\hline$c_{5}$ & 1 & $1.69314 \ldots$ & $1.69314 \ldots$ \\
\hline$c_{6}$ & $14.0617 \ldots$ & $20.7690 \ldots$ & $23.0016 \ldots$ \\
\hline$M_{0}$ & $2.90912 \times 10^{40}$ & $8.81788 \times 10^{145}$ & $5.88682 \times 10^{85}$ \\
\hline
\end{tabular}

which most likely will not be a lattice point. Here the relevant distance $d$ is that between $\mathbf{y}$ and the nearest lattice point, since now $\lambda$ defined by $\mathcal{A}\left(m_{1}^{\prime}, \ldots, m_{r}^{\prime}, m_{0}^{\prime}\right)^{\top}-$ $\mathbf{y}=\left(m_{1}^{\prime}, \ldots, m_{r}^{\prime}, \lambda\right)^{\top}$ is approximately $C L(P)$. To be precise, we have

$$
|\lambda-C L(P)| \leq \frac{1}{2}\left(r M_{0}+\left|m_{0}^{\prime}\right|+1\right) \leq r M_{0}+\frac{1}{2} .
$$


TABLE 15. Data of the reduction steps

\begin{tabular}{|l|l|llr|llr|lrr|}
\hline curve & $M_{0}$ & $C$ & $d>$ & $M_{1}$ & $C$ & $d>$ & $M_{2}$ & $C$ & $d>$ & $M_{3}$ \\
\hline Q24) & $2.90912 \times 10^{40}$ & $10^{125}$ & $2.42146 \times 10^{41}$ & 31 & $10^{7}$ & 231.400 & 9 & $10^{6}$ & 83.0301 & 8 \\
\hline (Q28) & $8.81788 \times 10^{145}$ & $10^{883}$ & $9.12503 \times 10^{146}$ & 52 & $10^{17}$ & 340.506 & 8 & $10^{12}$ & 52.0672 & 7 \\
\hline (Q48) & $5.88682 \times 10^{85}$ & $10^{347}$ & $2.66374 \times 10^{86}$ & 31 & $10^{10}$ & 123.292 & 6 & & & \\
\hline
\end{tabular}

TABLE 16. Runtimes

\begin{tabular}{|l|lll|}
\hline curve & comput. of $\phi\left(P_{i}\right)$ & reduction & small solut. \\
\hline (Q24) & $3 \mathrm{~s}$ & $<1 \mathrm{~s}$ & $5 \mathrm{~s}$ \\
(Q28) & $20 \mathrm{~m} 8 \mathrm{~s}$ & $16 \mathrm{~m} 56 \mathrm{~s}$ & $2 \mathrm{~h} 20 \mathrm{~m}$ \\
(Q48) & $1 \mathrm{~m} 2 \mathrm{~s}$ & $21 \mathrm{~s}$ & $3 \mathrm{~m} 43 \mathrm{~s}$ \\
\hline
\end{tabular}

With this taken into account, noting that the LLL-algorithm also provides a lower bound for this type of $d$, and using inequality (3), we get a reduced upper bound $M_{1}$ for $M$, namely

$$
M_{1}=\left\lfloor\sqrt{\frac{1}{c_{1}}\left(\log \left(c_{9} C\right)+\frac{1}{2} c_{10}+c_{11}-\log \left(\sqrt{d^{2}-r M_{0}^{2}}-\left(r M_{0}+\frac{1}{2}\right)\right)\right)}\right\rfloor
$$

We have reduction data as in Table 15 .

Again it is straightforward to find all solutions below the reduced bounds (although in the rank 5 case this takes some runtime). This completes the proof.

Finally we give in Table 16 the runtimes on a Pentium 75Mhz personal computer.

\section{The CASE "CUBIC = CUBIC"}

In this section we prove Theorem B36, and thus also Theorem A36. In order to do this we develop a variant of the elliptic logarithms method. See also [SW], where we hope to describe the method for solving any equation of type

$$
s_{1} U^{3}+s_{2} U^{2} V+s_{3} U V^{2}+s_{4} V^{3}+s_{5} U^{2}+s_{6} U V+s_{7} V^{2}+s_{8} U+s_{9} V+s_{10}=0
$$

that represents an elliptic curve. However, here we concentrate on the equation (C36) only, as a first example to gain experience with the method.

The birational transformations

$$
\left\{\begin{array} { l } 
{ U = \frac { 2 3 7 X + 1 5 Y - 3 3 7 5 } { 5 X ^ { 2 } + 4 5 X - 2 1 Y + 4 8 6 0 } , } \\
{ V = \frac { 3 1 5 X - 7 5 Y + 1 7 4 1 5 } { 5 X ^ { 2 } + 4 5 X - 2 1 Y + 4 8 6 0 } , }
\end{array} \quad \left\{\begin{array}{l}
X=\frac{45 U-75 V+300}{5 U+V} \\
Y=\frac{6750 U^{2}+1410 V^{2}+6300 U-4740 V}{(5 U+V)^{2}}
\end{array}\right.\right.
$$

relate equation (C36) and the minimal model

$$
Y^{2}=X^{3}-1575 X+52650 \text {. }
$$

See $[\mathrm{N}]$ and $[\mathrm{Co}$, Section 1.4] for an algorithm to compute these transformations.

In Table 17 some data on this elliptic curves are given, namely the minimal discriminant $\Delta$, the $j$-invariant $j$, and the torsion group.

Further we need the rank $r$, and a basis $P_{1}, \ldots, P_{r}$ for the free part of the Mordell-Weil group. We have the following result.

TABle 17. Data of elliptic curve

\begin{tabular}{|c|ccc|}
\hline curve & $\Delta$ & $j$ & torsion \\
\hline (W36) & $-947466720000=-2^{8} \cdot 3^{6} \cdot 5^{4} \cdot 8123$ & $3704400 / 8123=2^{4} \cdot 3^{3} \cdot 5^{2} \cdot 7^{3} \cdot 8123^{-1}$ & trivial \\
\hline
\end{tabular}


TABLE 18. Rank and basis of Mordell-Weil group

\begin{tabular}{|c|c|cccc|}
\hline curve & $r$ & $P_{1}$ & $P_{2}$ & $P_{3}$ & $P_{4}$ \\
\hline (W36) & 4 & $(-15,270)$ & $(15,180)$ & $(45,270)$ & $(-45,180)$ \\
\hline
\end{tabular}

TABLE 19. Relevant constants on heights of basis points

\begin{tabular}{|c|l|}
\hline curve & (W36) \\
\hline$B$ & 3.927 \\
$H$ & 12.73 \\
runtime & $1 \mathrm{~h} 27 \mathrm{~m}$ \\
\hline
\end{tabular}

Proposition 3. The elliptic curve (W36) has rank $r$ and basis $P_{1}, \ldots, P_{r}$ for the free part of its Mordell-Weil group as in Table 18.

Proof of Proposition 3. Again we use Cremona's mwrank. No difficulties were encountered. We give the constants $B$ and $H$ as well as the runtimes on a Sun Sparcstation 4 in Table 19.

Again note that the basis given is optimal for the least eigenvalue of the NéronTate height pairing matrix.

We consider a point

$$
P=m_{1} P_{1}+m_{2} P_{2}+m_{3} P_{3}+m_{4} P_{4}
$$

on the curve, with integral coordinates $U, V$ on $(\mathrm{C} 36)$. We put

$$
M=\max _{1 \leq i \leq 4}\left|m_{i}\right|
$$

As $\frac{d V}{3 U^{2}-1}$ and $\frac{d X}{Y}$ are two differential forms on the same elliptic curve, there should be a rational relationship. Indeed, a bit of calculation (see the birational transformations (4)) shows that

$$
\frac{d V}{3 U^{2}-1}=-\frac{15}{2} \frac{d X}{Y}
$$

For $(U, V) \in \mathbb{R}^{2}$ on (C36) with $V \geq 4$, we see that $U$ is a strictly increasing function of $V$, and likewise for $V \leq-1$. See Figure 1 .

For each point $(U, V) \in \mathbb{R}^{2}$ on (C36) with $V \leq-1$ or $V \geq 4$ there is a unique point $(X, Y) \in \mathbb{R}^{2}$ on (W36) with $Y \geq 0$, given by (4). For a point $P$ on the curve we use $(U, V)=(U(P), V(P))$ and $(X, Y)=(X(P), Y(P))$ to denote coordinates on both models. Let $F:(-\infty,-1] \cup[4, \infty) \longrightarrow \mathbb{R}$ be given by

$$
F(v)=15 \frac{3 u(v)-5 v+20}{5 u(v)+v}
$$

where $u(v)$ is the unique solution to $15 u(v)^{3}-15 u(v)=v^{3}-4 v^{2}+3 v$ for the given $v$. Then $X=F(V)$. 


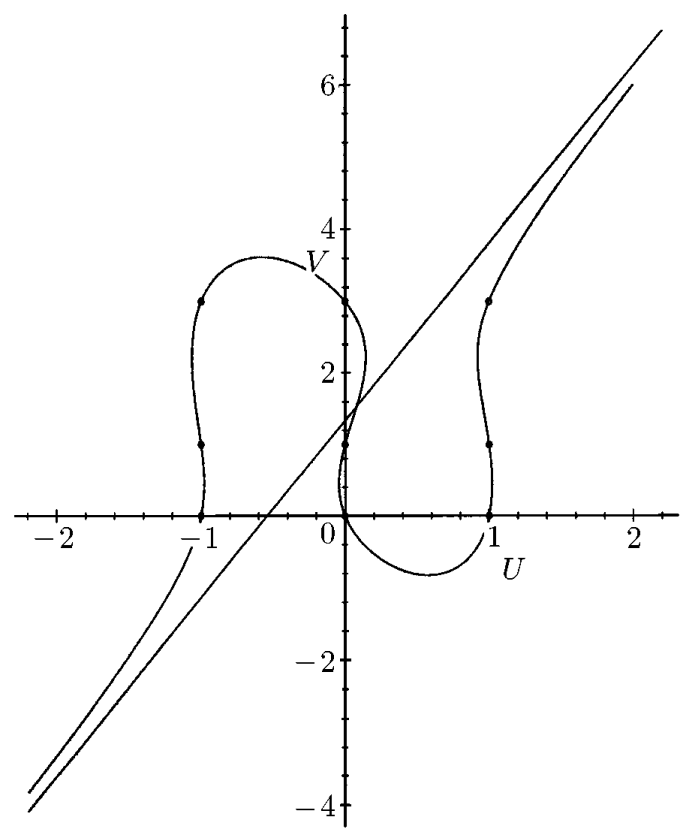

FIgURE 1. The elliptic curve (C36)

For $V \geq 4$ we have by (5) that

$$
\int_{V}^{\infty} \frac{d V}{3 U^{2}-1}=\frac{15}{2} \int_{X_{0}}^{X} \frac{d X}{Y}
$$

where

$$
X_{0}=\lim _{v \rightarrow \infty} F(v)=15 \frac{3-5 \alpha}{5+\alpha}=-15 \alpha+3 \alpha^{2},
$$

for $\alpha=\sqrt[3]{15}$. Let $Q_{0}=\left(-15 \alpha+3 \alpha^{2},-90+60 \alpha^{2}\right)$. Then $Q_{0} \in E(\mathbb{K})$, where $\mathbb{K}=\mathbb{Q}(\alpha)$, a cubic field, and $X_{0}=X\left(Q_{0}\right)$.

Likewise, for $V \leq-1$ we have by (5) that

$$
\int_{-\infty}^{V} \frac{d V}{3 U^{2}-1}=\frac{15}{2} \int_{X}^{X_{0}} \frac{d X}{Y}
$$

with $X_{0}$ as above. Note that $\alpha U-V+\frac{4}{3}=0$ is the asymptote of the curve (C36); see Figure 1.

For $\alpha U+\frac{4}{3}>V \geq 4$ or $\alpha U+\frac{4}{3}<V \leq-1$ we have $3 U^{2}-1 \geq\left(\frac{4}{45} \alpha-\frac{1}{16}\right) V^{2}$, so that in the case $V \geq 4$ we have

$$
\int_{V}^{\infty} \frac{d V}{3 U^{2}-1} \leq c \int_{V}^{\infty} \frac{d V}{V^{2}}=\frac{c}{V}
$$

and in the case $V \leq-1$ we have

$$
\int_{-\infty}^{V} \frac{d V}{3 U^{2}-1} \leq c \int_{-\infty}^{V} \frac{d V}{V^{2}}=\frac{c}{-V}
$$

where $c=\frac{45 \cdot 16}{64 \alpha-45}=6.38085 \ldots$ 
Now consider equation (W36). We have

$$
\begin{aligned}
\int_{X_{0}}^{X} \frac{d X}{Y} & =\int_{X_{0}}^{X} \frac{d X}{\sqrt{X^{3}-1575 X+52650}} \\
& =\int_{X_{0}}^{\infty} \frac{d X}{\sqrt{X^{3}-1575 X+52650}}-\int_{X}^{\infty} \frac{d X}{\sqrt{X^{3}-1575 X+52650}},
\end{aligned}
$$

and note that $X \in \mathbb{Q}$. Further,

$$
\begin{aligned}
\phi(P) & =\phi\left(m_{1} P_{1}+m_{2} P_{2}+m_{3} P_{3}+m_{4} P_{4}\right) \\
& =m_{1} \phi\left(P_{1}\right)+m_{2} \phi\left(P_{2}\right)+m_{3} \phi\left(P_{3}\right)+m_{4} \phi\left(P_{4}\right)+m_{0},
\end{aligned}
$$

with $m_{0} \in \mathbb{Z}$ such that all $\phi$-values are in $[0,1)$. By $(6)$ it follows in the case $V \geq 4$ that

$$
\begin{aligned}
\int_{V}^{\infty} \frac{d V}{3 U^{2}-1} & =\frac{15}{2} \int_{X_{0}}^{X} \frac{d X}{Y}=\frac{15}{2}\left(\omega \phi\left(Q_{0}\right)-\omega \phi(P)\right) \\
& =\frac{15}{2}\left(\omega \phi\left(Q_{0}\right)-m_{1} \omega \phi\left(P_{1}\right)-\ldots-m_{4} \omega \phi\left(P_{4}\right)-m_{0} \omega\right),
\end{aligned}
$$

where $\omega$ is the fundamental real period. Similarly, in the case $V \leq-1$ by (7) we have

$$
\begin{aligned}
\int_{-\infty}^{V} & \frac{d V}{3 U^{2}-1}=-\frac{15}{2} \int_{X_{0}}^{X} \frac{d X}{Y} \\
= & \frac{15}{2}\left(-\omega \phi\left(Q_{0}\right)+m_{1} \omega \phi\left(P_{1}\right)+\ldots+m_{4} \omega \phi\left(P_{4}\right)+m_{0} \omega\right) .
\end{aligned}
$$

Put $u_{0}=\omega \phi\left(Q_{0}\right)$ and $u_{i}=\omega \phi\left(P_{i}\right)$ for $i=1,2,3,4$, and let

$$
L(P)=u_{0}-m_{1} u_{1}-\ldots-m_{4} u_{4}-m_{0} \omega .
$$

Then (8), (9), (10) and (11) imply

$$
|L(P)|=\frac{2}{15} \int_{I} \frac{d V}{3 U^{2}-1} \leq \frac{2}{15} \frac{c}{|V|},
$$

with as integration interval $I=[V, \infty)$ if $V \geq 4$, and $I=(-\infty, V]$ if $V \leq-1$.

If $V \geq 6$ then $\alpha U<V<\alpha U+\frac{4}{3}$, and it follows that $X<0$. Moreover,

$$
15(-3 U+5 V-20)-(5 U+V)=74 V-50 U-300>\left(74-\frac{50}{\alpha}\right) V-300>0,
$$

so that the numerator $15(-3 U+5 V-20)$ of $|X|$ is larger than the denominator $5 U+V$.

Similarly, if $V \leq-5$ then $\alpha U+\frac{4}{3}<V<\alpha U+\alpha$, and it follows that $X<0$. Also,

$$
15(3 U-5 V+20)+(5 U+V)=-74 V+50 U+300>\left(\frac{50}{\alpha}-74\right) V+300>0,
$$

so that the numerator $15(3 U-5 V+20)$ of $|X|$ is larger than the denominator $-5 U-V$.

So if $V \geq 6$ or $V \leq-5$, then for the Weil height of $X$ we find

$$
\begin{aligned}
h(X) & \leq \log (15|3 U-5 V+20|)<\log \left(15\left(\left(5-\frac{3}{\alpha}\right)|V|+\operatorname{sign}(V)\left(\frac{4}{\alpha}-20\right)\right)\right) \\
& \leq \log \left(135-\frac{57}{\alpha}\right)+\log |V|<4.71750+\log |V| .
\end{aligned}
$$


TABLE 20. Relevant constants

\begin{tabular}{|r|l|}
\hline curve & $(\mathrm{C} 36)$ \\
\hline$\hat{h}\left(P_{1}\right)$ & $0.561125 \ldots$ \\
$\hat{h}\left(P_{2}\right)$ & $0.566020 \ldots$ \\
$\hat{h}\left(P_{3}\right)$ & $0.578280 \ldots$ \\
$\hat{h}\left(P_{4}\right)$ & $0.736397 \ldots$ \\
$\hat{h}\left(Q_{0}\right)<$ & 5.66238 \\
$c_{1}$ & $0.384689 \ldots$ \\
$\phi\left(P_{1}\right)$ & $0.383460 \ldots$ \\
$\phi\left(P_{2}\right)$ & $0.298105 \ldots$ \\
$\phi\left(P_{3}\right)$ & $0.198210 \ldots$ \\
$\phi\left(P_{4}\right)$ & $0.459223 \ldots$ \\
\hline
\end{tabular}

\begin{tabular}{|r|l|}
\hline curve & $(\mathrm{C} 36)$ \\
\hline$\phi\left(Q_{0}\right)$ & $0.392231 \ldots$ \\
$u_{1}$ & $0.600689 \ldots$ \\
$u_{2}$ & $0.466981 \ldots$ \\
$u_{3}$ & $0.310495 \ldots$ \\
$u_{4}$ & $0.719371 \ldots$ \\
$u_{0}$ & $0.614429 \ldots$ \\
$h_{E}$ & $15.1250 \ldots$ \\
$D$ & 3 \\
$A_{0}$ & $15.1250 \ldots$ \\
\hline
\end{tabular}

\begin{tabular}{|r|l|}
\hline curve & $(\mathrm{C} 36)$ \\
\hline$A_{1}$ & $15.1250 \ldots$ \\
$A_{2}$ & $15.1250 \ldots$ \\
$A_{3}$ & $15.1250 \ldots$ \\
$A_{4}$ & $15.1250 \ldots$ \\
$A_{0}^{\prime}$ & $15.1250 \ldots$ \\
$c_{4}$ & $2.79031 \times 10^{216}$ \\
$c_{5}$ & $2.09861 \ldots$ \\
$c_{6}$ & $17.2236 \ldots$ \\
$M_{0}$ & $1.75995 \times 10^{114}$ \\
\hline
\end{tabular}

It is noteworthy that the above formula is the only point in this proof where we use the fact that $U, V$ are integral.

From Silverman [Sil2] we have

$$
\hat{h}(P)-\frac{1}{2} h(X)<3.87831,
$$

and moreover

$$
\hat{h}(P) \geq c_{1} M^{2} .
$$

Putting everything together, we obtain from (12), (13), (14) and (15), under the condition $V \geq 6$ or $V \leq-5$, that

$$
|L(P)|<\frac{2}{15} \frac{c}{|V|}<e^{12.3126-2 c_{1} M^{2}} .
$$

Now we apply David's result [D], and obtain

$$
|L(P)|>\exp \left(-c_{4}\left(\log (4 M)+c_{5}\right)\left(\log \log (4 M)+c_{6}\right)^{7}\right) .
$$

Together with (16) this yields an absolute upper bound $M_{0}$ for $M$. We have the data as in Table 20. Note that we used that $\left|m_{0}\right| \leq 4 M$.

The reduction procedure runs just as in the previous section for the cases of (Q28) and (Q48), since the linear form also is inhomogeneous in this case. Thus the lattice is spanned by the columns of

$$
\mathcal{A}=\left(\begin{array}{ccccc}
1 & 0 & 0 & 0 & 0 \\
0 & 1 & 0 & 0 & 0 \\
0 & 0 & 1 & 0 & 0 \\
0 & 0 & 0 & 1 & 0 \\
{\left[C u_{1}\right]} & {\left[C u_{2}\right]} & {\left[C u_{3}\right]} & {\left[C u_{4}\right]} & {[C \omega]}
\end{array}\right)
$$

and $d$ is the distance between the point

$$
\mathbf{y}=\left(0,0,0,0,-\left[C u_{0}\right]\right)^{\top}
$$

and the nearest lattice point. We have

$$
|\lambda-C L(P)| \leq \frac{1}{2}\left(4 M_{0}+\left|m_{0}^{\prime}\right|+1\right) \leq 4 M_{0}+\frac{1}{2},
$$


TABLE 21. Data of the reduction steps for (C36)

\begin{tabular}{|l|l|llr|llr|}
\hline curve & $M_{0}$ & $C$ & $d>$ & $M_{1}$ & $C$ & $d>$ & $M_{2}$ \\
\hline (C36) & $1.75995 \times 10^{114}$ & $10^{575}$ & $1.12582 \times 10^{115}$ & 37 & $10^{13}$ & 265.465 & 6 \\
\hline
\end{tabular}

TABLE 22. Runtimes

\begin{tabular}{|l|lll|}
\hline curve & comput. of $\phi\left(P_{i}\right)$ & reduction & small solut. \\
\hline (C36) & $4 \mathrm{~m} 38 \mathrm{~s}$ & $2 \mathrm{~m} 44 \mathrm{~s}$ & $9 \mathrm{~m} 19 \mathrm{~s}$ \\
\hline
\end{tabular}

and thus, using inequality (16), we reach a reduced upper bound $M_{1}$ for $M$, namely

$$
M_{1}=\left\lfloor\sqrt{\frac{1}{2 c_{1}}\left(\log C+12.3126-\log \left(\sqrt{d^{2}-4 M_{0}^{2}}-\left(4 M_{0}+\frac{1}{2}\right)\right)\right)}\right\rfloor .
$$

We have reduction data as in Table 21.

Again it is straightforward to find all solutions below the reduced bound. This completes the proof.

Finally we give in Table 22 the runtimes on a Pentium $75 \mathrm{Mhz}$ personal computer.

\section{REFERENCES}

[A] È.T. Avanesov, "Solution of a problem on figurative numbers" (Russian), Acta Arithmetica 12 [1966/67], 409-420. MR 35:6619

[BC] A. Baker and J. Coates, "Integer points on curves of genus 1", Proc. Camb. Phil. Soc. 67 [1970], 595-602. MR 41:1638

[BH] Yu. F. Bilu and G. Hanrot, "Solving superelliptic Diophantine equations by Baker's method", Compositio Math. 112 (3) [1998], 223-312.

[Co] I. Connell, The elliptic curve handbook, manuscript, 1997. Available from the ftp site of McGill University in the directory math.mcgill.ca/pub/ECH1. From the same site the Apecs package can be downloaded.

[Cr] J.E. Cremona, Algorithms for modular elliptic curves, Cambridge University Press, Cambridge, 1992. MR 93m:11053 The programs mrank, mwrank, findinf may be downloaded from John Cremona's ftp site euclid.ex.ac.uk/pub/cremona.

[D] S. David, Minorations de formes linéaires de logarithmes elliptiques, Mém. Soc. Math. France, Vol. 62, 1995. MR 98f:11078

[F] G. Faltings, "Endlichkeitssätze für abelsche Varietäten über Zahlkörpern", Invent. Math. 73 [1983], 349-366. MR 85g:11026a

[GPZ] J. Gebel, A. Pethő and H.G. Zimmer, "Computing integral points on elliptic curves", Acta Arithmetica 68 [1994], 171-192. MR 95i:11020

[L] D.A. LIND, "The quadratic field $\mathbb{Q}(\sqrt{5})$ and a certain diophantine equation", Fibonacci Quarterly 6 [1968], 86-93. MR 38:112

[M1] L.J. Mordell, "On the integer solutions of $y(y+1)=x(x+1)(x+2)$ ", Pacific Journal of Mathematics 13 [1963], 1347-1351. MR 27:3590

[M2] L.J. Mordell, Diophantine Equations, Academic Press, London, New York, 1969. MR 40:2600

[N] T. NAGell, "Sur les propriétés arithmétiques des cubiques planes du premier genre", Acta Mathematica 52 [1928/9], 93-126.

[P] Á. Pintér, "A note on the diophantine equation $\left(\begin{array}{l}x \\ 4\end{array}\right)=\left(\begin{array}{l}y \\ 2\end{array}\right)$ ", Publ. Math. Debrecen 47 [1995], 411-415. MR 96i:11027

[Sik] S. Siksek, "Infinite descent on elliptic curves", Rocky Mountain J. Math. 25 [1995], 15011538. MR 97g: 11053

[Sil1] J.H. Silverman The arithmetic of elliptic curves, Springer Verlag, Berlin etc., 1986. MR 87g:11070 
[Sil2] J.H. Silverman, "The difference between the Weil height and the canonical height on elliptic curves", Math. Comput. 55 [1990], 723-743. MR 91d:11063

[Sin] D. Singmaster, "Repeated binomial coefficients and Fibonacci numbers", Fibonacci Quarterly 13 [1975], 295-298. MR 54:224

[Sm] N.P. Smart, "S-integral points on elliptic curves", Mathematical Proceedings of the Cambridge Philosophical Society 116 [1994], 391-399. MR 95g:11050

[ST1] R.J. Stroeker and N. Tzanakis, "Solving elliptic diophantine equations by estimating linear forms in elliptic logarithms", Acta Arithmetica 67 [1994], 177-196. MR 95m:11056

[ST2] R.J. Stroeker and N. Tzanakis, "On the Elliptic Logarithm Method for Elliptic Diophantine Equations. Reflections and an Improvement", to appear in Experimental Math.

[SW] R.J. Stroeker and B.M.M. De Weger, "Solving Elliptic Diophantine Equations: The General Cubic Case", submitted to Acta Arithmetica.

[T] N. Tzanakis, "Solving elliptic diophantine equations by estimating linear forms in ellitpic logarithms. The case of quartic equations", Acta Arithmetica 75 [1996], 165-190. MR 96m: 11019

[TW] N. Tzanakis and B.M.M. DE Weger, "On the practical solution of the Thue equation", J. Number Th. 31 [1989], 99-132. MR 90c:11018

[dW1] B.M.M. DE Weger, "A binomial diophantine equation", Quarterly Journal of Mathematics 47 [1996], 221-231. MR 97c:11041

[dW2] B.M.M. DE WEGER, "Equal binomial coefficients: some elementary considerations", Journal of Number Theory 63 [1997], 373-386. MR 98b:11027

Econometric Institute, Erasmus University Rotterdam, P.O. Box 1738, 3000 DR RotTERDAM, THE NetherLANDS

E-mail address: stroeker@few.eur.nl

Sportsingel 30, 2924 XN Krimpen aAn den ijssel, The Neterlands

E-mail address: deweger@xs4all.nl 